\title{
QUANTUM COADJOINT ACTION
}

\author{
C. DE CONCINI, V. G. KAC, AND C. PROCESI
}

\section{INTRODUCTION AND NOTATIONS}

0.1 . This paper is a continuation of the paper [DCK] on representations of quantum groups at roots of 1 . We give a solution to most of the conjectures stated in [DCK, §5] on the center and on the quantum coadjoint action (some of the conjectures needed modification to be correct).

As in the case of Lie groups, "simply connected" quantum groups are nicer than the more popular "adjoint" quantum groups. The primary object of the present paper is the simply connected quantum group, the adjoint quantum group being the subalgebra of invariants of the center of the corresponding simply connected Lie group.

The classical orbit method relates representations of a Lie group to the orbits of the coadjoint action of this group in the dual of the Lie algebra. The basic observation of the present paper is that representations of a quantum group at roots of 1 are closely related to the orbits of the action of the corresponding group on itself by conjugation.

0.2 . Let us first introduce the necessary notations. Fix an $n \times n$ indecomposable matrix $\left(a_{i j}\right)$ with integer entries such that $a_{i i}=2$ and $a_{i j} \leq 0$ for $i \neq j$ and a vector $\left(d_{1}, \ldots, d_{n}\right)$ with relatively prime positive integral entries $d_{i}$ such that the matrix $\left(d_{i} a_{i j}\right)$ is symmetric and positive definite.

Let $P$ be a free abelian group with basis $\omega_{i}, i=1, \ldots, n$, and let

$$
\rho=\sum_{i=1}^{n} \omega_{i}, \quad \alpha_{j}=\sum_{i=1}^{n} a_{i j} \omega_{i} \quad(j=1, \ldots, n) .
$$

Let $Q=\sum_{i} \mathbb{Z} \alpha_{i}, Q_{+}=\sum_{i} \mathbb{Z}_{+} \alpha_{i}$. For $\beta=\sum_{i} k_{i} \alpha_{i} \in Q$ let ht $\beta=\sum_{i} k_{i}$ be the height of $\beta$.

Define a bilinear pairing $P \times Q \rightarrow \mathbb{Z}$ by $\left(\omega_{i} \mid \alpha_{j}\right)=\delta_{i j} d_{j}$. Then $\left(\alpha_{i} \mid \alpha_{j}\right)=$ $d_{i} a_{i j}$, giving a symmetric $\mathbb{Z}$-valued bilinear form on $Q$ such that $(\alpha \mid \alpha) \in 2 \mathbb{Z}$. Note also that $2\left(\omega_{i} \mid \rho\right) \in \mathbb{Z}$ since $2 \rho \in Q$.

Define automorphisms $s_{i}$ of $P$ by $s_{i}\left(\omega_{j}\right)=\omega_{j}-\delta_{i j} \alpha_{i}(i, j=1, \ldots, n)$. Then $s_{i}\left(\alpha_{j}\right)=\alpha_{j}-a_{i j} \alpha_{i}$. Let $W$ be the (finite) subgroup of $\operatorname{GL}(P)$ generated

Received by the editors January 8, 1991, and, in revised form, July 11, 1991.

1991 Mathematics Subject Classification. Primary 17B37, 81R50.

Supported in part by NSF grant DMS-8802489 and by DOE grant DE-FG02-88ER25066. 
by $s_{1}, \ldots, s_{n}$. Then $Q$ is $W$-invariant and the pairing $P \times Q \rightarrow \mathbb{Z}$ is $W$ invariant. Let

$$
\Pi=\left\{\alpha_{1}, \ldots, \alpha_{n}\right\}, \quad R=W \Pi, \quad R^{+}=R \cap Q_{+} .
$$

Then, of course, $R$ is the root system corresponding to the Cartan matrix $\left(a_{i j}\right)$, $Q$ is the root lattice, $W$ is the Weyl group, $R^{+}$is a set of positive roots, $\Pi$ the corresponding set of simple roots, etc.

Given a lattice $M$, we denote as usual by $M^{*}=\operatorname{Hom}_{\mathbb{Z}}(M, \mathbb{Z})$ the dual lattice. For example, $P^{*}$ may be identified, using the bilinear form $(\cdot \mid \cdot)$, with the coroot lattice $Q^{\vee}=\sum_{i} \mathbb{Z} \alpha_{i}^{\vee}$, where $\alpha_{i}^{\vee}=d_{i}^{-1} \alpha_{i}$.

0.3 . Let $q$ be an indeterminate and let $q_{i}=q^{d_{i}}$. The simply connected quantum group is the $\mathbb{C}(q)$-algebra $U_{P}$ on generators $E_{i}, F_{i}, L_{i}, L_{i}^{-1}(1 \leq i \leq n)$ and the following defining relations:

(1) $L_{i} L_{j}=L_{j} L_{i}, L_{i} L_{i}^{-1}=L_{i}^{-1} L_{i}=1$;

(2) $L_{i} E_{j} L_{i}^{-1}=q_{i}^{\delta_{i j}} E_{j}, L_{i} F_{j} L_{i}^{-1}=q_{i}^{-\delta_{i j}} F_{j}$;

(3) $E_{i} F_{j}-F_{j} E_{i}=\delta_{i j}\left(K_{i}-K_{i}^{-1}\right) /\left(q_{i}-q_{i}^{-1}\right)$,

where for $\beta=\sum m_{i} \omega_{i} \in P$ we let $K_{\beta}=\prod_{j} L_{j}^{m_{j}}$, and let $K_{i}=K_{\alpha_{i}}$;

(4) certain Chevalley type relations between the $E_{i}$ and between the $F_{i}$ (see, e.g., [DCK, (1.2.4 and 5)]).

The quantum group of Drinfeld-Jimbo is the subalgebra of $U_{P}$ over $\mathbb{C}(q)$ generated by the $E_{i}, F_{i}, K_{i}, K_{i}^{-1}(i=1, \ldots, n)$. More generally, for any lattice $M$ between $P$ and $Q$ one may consider the intermediate quantum group $U_{M}$ generated by the $E_{i}, F_{i}(i=1, \ldots, n)$ and the $K_{\beta}$ with $\beta \in M$. In this paper by a quantum group we mean one of these algebras. We denote by $U^{+}, U^{-}$, and $U^{0}$ the $\mathbb{C}(q)$-subalgebra of $U_{M}$ generated by the $E_{i}$, the $F_{i}$, and the $K_{\beta}$ respectively, and by $\bar{U}^{+}$and $\bar{U}^{-}$the two sided ideals of $U^{+}$and $U^{-}$generated by the $E_{i}$ and the $F_{i}$ respectively. We shall sometimes add the subscript $M$ to emphasize the dependence on $M$, like $U_{M}^{0}$, etc.

0.4. As usual, for $n \in \mathbb{Z}$ and $d \in \mathbb{N}$ we let

$$
[n]_{d}=\left(q^{d n}-q^{-d n}\right) /\left(q^{d}-q^{-d}\right), \quad[n]_{d} !=[1]_{d}[2]_{d} \cdots[n]_{d} .
$$

Given $s \in \mathbb{N}$ we shall write $E_{i}^{(s)}$ and $F_{i}^{(s)}$ for $E_{i}^{s} /[s]_{d_{i}}$ ! and $F_{i}^{s} /[s]_{d_{i}}$ ! respectively.

Due to [L] we have an action of the braid group $\mathscr{B}$ with generators $T_{1}, \ldots$, $T_{n}$ (see $\S 2.1$ for its definition) by automorphisms of $U_{M}$ defined as follows:

$$
\begin{gathered}
T_{i} E_{i}=-F_{i} K_{i}, \quad T_{i} E_{j}=\sum_{s=0}^{-a_{i j}}(-1)^{s-a_{i j}} q_{i}^{-s} E_{i}^{\left(-a_{i j}-s\right)} E_{j} E_{i}^{(s)} \quad \text { if } i \neq j, \\
T_{i} F_{i}=-K_{i}^{-1} E_{i}, \quad T_{i} F_{j}=\sum_{s=0}^{-a_{i j}}(-1)^{s-a_{i j}} q_{i}^{-s} F_{i}^{(s)} F_{j} F_{i}^{\left(-a_{i j}-s\right)} \text { if } i \neq j, \\
T_{i} K_{\beta}=K_{s_{i} \beta} .
\end{gathered}
$$


0.5 . Now let $l$ be an odd integer greater than 1 and relatively prime to all the $d_{i}$ (this condition, imposed throughout the paper, comes from the use of formulas in [L, DCK]), and let $\varepsilon$ be a primitive $l$ th root of 1 . We denote by $U_{M, \varepsilon}$ the algebra over $\mathbb{C}$ obtained from $U_{M}$ by specializing $q$ to $\varepsilon$, and by $U_{\varepsilon}^{+}, U_{\varepsilon}^{-}$, etc. the specializations of the subalgebras $U^{+}, U^{-}$, etc. More precisely, we let $\mathscr{A}=\mathbb{C}\left[q, q^{-1}\right]$ and denote by $U_{M, \mathscr{A}}$ the $\mathscr{A}$-subalgebra of $U_{M}$ generated by the $E_{i}, F_{i}, K_{\beta}$, and $\left(K_{i}-K_{i}^{-1}\right) /\left(q_{i}-q_{i}^{-1}\right)$; then $U_{M, \varepsilon}=U_{M, \mathscr{A}} /(q-\varepsilon)$, etc.

Let $c_{i}=\left(\varepsilon^{d_{i}}-\varepsilon^{-d_{i}}\right)^{l}$ and consider the elements

$$
y_{i}=c_{i} F_{i}^{l}, \quad z_{\beta}=K_{\beta}^{l} \quad(\beta \in M), \quad z_{i}=z_{\alpha_{i}} .
$$

These elements lie in the center $Z_{\varepsilon}$ of $U_{M, \varepsilon}$ [DCK, $\left.\S 3.1\right]$. Denote by $Z_{0}$ the smallest $\mathscr{B}$-invariant subalgebra of $Z_{\varepsilon}$ containing all these elements.

Our first main result is a description of the $\mathscr{B}$-algebra $Z_{0}$ by generators and relations $(\S 3.5)$. In the simply laced case, i.e., when $\left(a_{i j}\right)$ is symmetric, the result is:

Let $Z_{0}^{0}$ be the subalgebra of $Z_{0}$ spanned by the $z_{\beta} \quad(\beta \in M)$ with the $\mathscr{B}$-action given by $T_{i} z_{\beta}=z_{s_{i} \beta}$. Then $Z_{0}$ is a commutative $\mathscr{B}$-algebra over the $\mathscr{B}$-algebra $Z_{0}^{0}$ on generators $T y_{i}(T \in \mathscr{B}, i=1, \ldots, n)$ and defining relations

(1) $T_{i}^{2} y_{i}=z_{i}^{2} y_{i}$

(2) $T_{i} y_{j}=y_{j}$ if $a_{i j}=0$,

(3a) $T_{i} T_{j} y_{i}=y_{j}$ if $a_{i j}=-1$,

(3b) $T_{i} y_{j}+T_{j} y_{i}=y_{i} y_{j}$ if $a_{i j}=-1$.

It is interesting to note a close connection of this with the well-known action of $\mathscr{B}$ on the simple Lie algebra $\mathfrak{g}$ associated to the matrix $\left(a_{i j}\right)$ (Proposition 4.2):

Let $D$ be the smallest $\mathscr{B}$-invariant subalgebra in $S(\mathfrak{g})$ (the symmetric algebra over $\mathfrak{g}$ ) containing the Chevalley generators $f_{1}, \ldots, f_{n}$ (root vectors attached to negative simple roots). Then $D$ is a commutative $\mathscr{B}$-algebra over $\mathbb{C}$ on generators $T f_{i}(T \in \mathscr{B}, i=1, \ldots, n)$ and the "homogenized" defining relations of $Z_{0}$

$$
\begin{gathered}
T_{i}^{2} f_{i}=f_{i}, \quad T_{i} f_{j}=f_{j} \quad \text { if } a_{i j}=0, \\
T_{i} T_{j} f_{i}=f_{j} \quad \text { and } \quad T_{i} f_{j}+T_{j} f_{i}=0 \quad \text { if } a_{i j}=-1 .
\end{gathered}
$$

0.6 . Let $G$ be the connected complex Lie group whose Lie algebra is $\mathfrak{g}$ and such that any maximal complex torus of $G$ has character group $M$ (so that Center $G=M / Q$ ). Let $T$ be a maximal torus of $G$, and let $U_{+}$(resp. $U_{-}$) be the maximal unipotent subgroup of $G$ corresponding to $R^{+}$(resp. to $-R^{+}$). Note that $T=\operatorname{Spec} Z_{0}^{0}$ with the usual $W$-action.

Our main construction is the map $\pi$ of $\Omega_{M}:=\operatorname{Spec} Z_{0}$ to the "big cell" $G^{0}:=U_{-} T U_{+}$constructed as follows. Fix a reduced expression $J$ of the longest element of $W: w_{0}=s_{i_{1}} s_{i_{2}} \cdots s_{i_{N}}$. Let $T_{k-1}^{J}=T_{i_{1}} \cdots T_{i_{k-1}}(k=1, \ldots, N)$, 
$T_{0}=T_{N}^{J}$, and consider the elements

$$
y_{k}^{J}=T_{k-1}^{J} y_{i_{k}} \in Z_{0}, \quad f_{k}^{J}=T_{k-1}^{J} f_{i_{k}} \in \mathfrak{g} .
$$

Let $Z_{0}^{-}$(resp. $Z_{0}^{+}$) be the subalgebra generated by the $y_{k}^{J}\left(\right.$ resp. $\left.T_{0} y_{k}^{J}\right), k=$ $1, \ldots, N$. These are polynomial algebras (independent of the choice of $J$; see $\S 3.3)$ and we have

$$
Z_{0}=Z_{0}^{-} \otimes Z_{0}^{0} \otimes Z_{0}^{+},
$$

so that $\Omega_{M} \simeq \mathbb{C}^{N} \times T \times \mathbb{C}^{N}$ (noncanonically). Define the maps

$$
Y: \operatorname{Spec} Z_{0}^{-} \rightarrow U_{-}, \quad X: \operatorname{Spec} Z_{0}^{+} \rightarrow U_{+}, \quad Z: T \rightarrow T
$$

as

$$
\begin{gathered}
Y=\left(\exp y_{N}^{J} f_{N}^{J}\right)\left(\exp y_{N-1}^{J} f_{N-1}^{J}\right) \cdots\left(\exp y_{1}^{J} f_{1}^{J}\right), \quad X=T_{0}(Y), \\
Z(t)=t^{2}, \quad t \in T .
\end{gathered}
$$

Here $\mathscr{B}$ acts in the obvious way: $T_{i}(\cdots \exp y f \cdots)=\cdots \exp \left(\left(T_{i} y\right)\left(T_{i} f\right)\right) \cdots$, $y \in Z_{0}, f \in \mathfrak{g}$, where its action on $\mathfrak{g}$ is the usual one (see $\S 4.1$ ). We show that the maps $Y$ and $X$ are independent of the choice of $J$, and we let

$$
\pi=Y Z X: \Omega_{M} \rightarrow G^{0} .
$$

This is an unramified cover of degree $2^{n}$.

In $\S 4$ we study the interplay between the map $\pi$ and the action of the braid group $\mathscr{B}$ on $\Omega_{M}$ and on $G$.

0.7. The primary object of our study is the quantum coadjoint action defined as follows [DCK]. We have derivations $\underline{e}_{i}$ and $\underline{f}_{i}$ of $U_{M, \varepsilon}(i=1, \ldots, n)$ defined by

$$
\underline{e}_{i}(u)=\lim _{q \mapsto \varepsilon}\left[E_{i}^{(l)}, u\right], \quad \underline{f}_{i}=T_{i} \underline{e}_{i} T_{i}^{-1} .
$$

We denote by $\widetilde{G}$ the (infinite-dimensional) group of analytic automorphisms of the variety $\Omega_{M}$ generated by the 1-parameter groups $\exp t \underline{e}_{i}$ and $\exp t \underline{f}_{i}$ $(t \in \mathbb{C}, i=1, \ldots, n)$.

The key calculations of the paper are the following formulas relating the Chevalley generators $e_{i}$ and $f_{i}$ to the derivations $\underline{e}_{i}$ and $\underline{f}_{i}$ (Theorems 5.4 and 5.5)

$$
\underline{e}_{i}=z_{i} f_{i}, \quad \underline{f}_{i}=-z_{i} e_{i} .
$$

Here by $e_{i}$ and $f_{i}$ we understand the pullback via the covering $\pi$ of the Killing vector fields on $G$ defined by the $e_{i}, f_{i} \in \mathfrak{g}$.

The proof of the formulas (!) is rather straightforward, but requires heavy computations. For simplicity of the exposition, we present all details of the calculation in the simply laced case, leaving out the details in other cases.

We use these formulas to describe the orbits of the group $\widetilde{G}$ on $\Omega_{M}(\S 6)$. In order to state the result we need one more construction.

Let $G_{c}$ be the simply connected cover of $G$, so that $G=G_{c} / C$, where $C \simeq P / M$. We let $G^{\prime}=G_{c} / C^{2}$. This is an unramified $C / C^{2}$-cover $\phi: G^{\prime} \rightarrow G$ 
of $G$. The map $\pi$ factors through $G^{\prime}$, i.e., there exists a unique unramified cover $\pi^{\prime}: \Omega_{M} \rightarrow G^{\prime 0}$ such that $\pi=\phi \circ \pi^{\prime}$.

Let $\mathscr{O}$ be a conjugacy class in $G^{\prime}$ of a noncentral element and let $\mathscr{O}^{0}=$ $\mathscr{O} \cap G^{\prime 0}$. We show that $\left(\pi^{\prime}\right)^{-1} \mathscr{O}^{0}$ is a $\widetilde{G}$-orbit in $\Omega_{M}$ and these are all orbits of nonfixed points of $\widetilde{G}$; all the fixed points are points of the fibers of $\pi^{\prime}$ over central elements of $G^{\prime}$ (Theorem 6.6).

The study of the orbits of $\widetilde{G}$ on $\operatorname{Spec} Z_{\varepsilon}$ and $\operatorname{Spec} Z_{0}$ is important for the following reason [DCK]. Let $\operatorname{Spec} U_{M, \varepsilon}$ denote the set of all equivalence classes of finite-dimensional irreducible representations of $U_{M, \varepsilon}$. The group $\widetilde{G}$ acts on Spec $U_{M, \varepsilon}$ in a natural way. Associating to an irreducible representation its central character gives a surjective $\widetilde{G}$-equivariant map $\chi: \operatorname{Spec} U_{M, \varepsilon} \rightarrow \operatorname{Spec} Z_{\varepsilon}$ that is generically bijective. The inclusion $Z_{0} \subset Z_{\varepsilon}$ induces a finite (hence surjective) map $\tau: \operatorname{Spec} Z_{\varepsilon} \rightarrow \operatorname{Spec} Z_{0}$. Thus, we obtain a sequence of canonical surjective maps

$$
\operatorname{Spec} U_{M, \varepsilon} \stackrel{\chi}{\longrightarrow} \operatorname{Spec} Z_{\varepsilon} \stackrel{\tau}{\longrightarrow} \operatorname{Spec} Z_{0} .
$$

Representations from the same $\widetilde{G}$-orbit in $\operatorname{Spec} U_{M, \varepsilon}$ are practically the same. Thus in order to describe $\operatorname{Spec} U_{M, \varepsilon}$, it suffices for each orbit to describe the fiber of $\chi$. The structure of representations from the fiber should be intimately related to geometric properties of the orbit (see, e.g., Conjecture 6.8).

The first important application of the description of the orbits of $\widetilde{G}$ in $\Omega_{M}$ is the "triangulizability" of any $\pi \in \operatorname{Spec} U_{M, \varepsilon}:$ there exists an automorphism $\sigma$ and a nonzero vector $v$ in the representation space of $\pi$ such that $\pi\left(\sigma\left(E_{i}\right)\right) v=$ 0 for all $i$.

0.8. Denote by $Q_{2}^{\vee}$ the subgroup of $T$ consisting of elements $\exp \pi i \alpha, \alpha \in$ $Q^{\vee} \subset M^{*}$, and let $\widetilde{W}=W \ltimes Q_{2}^{\vee}$. It follows from $\S 0.7$ that two nonfixed points of $T \subset \Omega_{M}$ lie in the same orbit of $\widetilde{G}$ if and only if they lie in the same orbit of $\widetilde{W}$. This leads to a Chevalley type restriction theorem (Theorem 6.7):

The restriction homomorphism induced by inclusion $T \subset \Omega_{M}$ gives an isomorphism of algebras of invariants: $Z_{0}^{\widetilde{G}} \stackrel{\sim}{\rightarrow} Z_{0}^{0 \widetilde{W}}$. (One can actually show that this extends to an isomorphism $Z_{\varepsilon}^{\widetilde{G}} \stackrel{\sim}{\rightarrow} U_{\varepsilon}^{0 \widetilde{W}}$.)

0.9. Let $Z$ be the center of the quantum group $U_{M}$. In [DCK] an explicit isomorphism $\bar{h}: U^{0 \widetilde{W}} \stackrel{\sim}{\rightarrow} Z$ was constructed (only the case $M=Q$ was considered there, but the construction and the proof extend to arbitrary $M)$. We show that $\bar{h}$ can be specialized to $q=\varepsilon$ to give an injective homomorphism $\bar{h}_{\varepsilon}: U_{\varepsilon}^{0 \widetilde{W}} \rightarrow Z_{\varepsilon}$ (Proposition 6.2). We denote the image of this homomorphism by $Z_{1}$. One can show in fact that $Z_{1}=Z_{\varepsilon}^{\widetilde{G}}$ (Theorem $\left.6.7(\mathrm{c})\right)$.

Our next important result (Theorem 6.4(a)) states that the subalgebras $Z_{P, 0}$ and $Z_{P, 1}$ generate the center $Z_{P, \varepsilon}$ (for arbitrary $M$ this may be false). The proof of this theorem is roughly as follows. Consider the subalgebra $\widetilde{Z}_{M, \varepsilon}$ generated by $Z_{0}$ and $Z_{1}$. It is easy to see for arbitrary $M$ that the quotient 
fields of $\tilde{Z}_{M, \varepsilon}$ and $Z_{M, \varepsilon}$ coincide. Also it was shown in [DCK] that $Z_{M, \varepsilon}$ is integrally closed (this again holds for arbitrary $M$ ). Thus, we have to show that $\widetilde{Z}_{P, \varepsilon}$ is integrally closed. We show that $\widetilde{Z}_{P, \varepsilon}$ is a complete intersection ring that is smooth in codimension one and hence (by Serre's theorem) is integrally closed. We therefore deduce that $\operatorname{Spec} Z_{P, \varepsilon}$ is a complete intersection (Theorem 6.4(b)).

At the same time, we obtain a simple geometric construction of the center $Z_{P, \varepsilon}$. Let $G$ be simply connected. Denote by $\rho_{l}$ the map $g \mapsto g^{l}$ of $G$ into itself. Let $\mathbb{C}[G]^{G}$ be the algebra of invariant polynomials on $G$ under conjugation, let $G / / G$ denote the corresponding affine variety, and let $\sigma: G \rightarrow$ $G / / G$ be the quotient map. Then $\operatorname{Spec} Z_{P, \varepsilon}$ is an unramified cover with the Galois group $\frac{1}{2} Q^{\vee} / Q^{\vee}$ of the fiber product

$$
(G / / G) \times_{G / / G} G^{0}
$$

where the first map is induced by $\rho_{l}$ and the second map is the restriction of $\sigma$ to the big cell. We show that this fiber product may be obtained using the Stein factorization of the $l$ th power map in $G(\S 6.5)$. Note that this is the only instance where $l$ enters in the geometric picture. We derive from this result a description of the action of $\widetilde{G}$ on $\operatorname{Spec} Z_{P, \varepsilon}$ and of the fibers of the map $\tau$ (Corollary 6.4).

0.10 . In $\S 7$ we interpret our results in the language of Poisson algebraic groups. Explicitly, we show that $\Omega_{M}$ has a canonical structure of a Poisson algebraic group, which turns out to be isomorphic to the dual of the celebrated SklyaninDrinfeld Poisson Lie group. The $\widetilde{G}$-orbits are precisely the symplectic leaves of this Poisson structure. The map $\pi$ and the action of $\mathscr{B}$ have a simple description in this framework.

It is worth mentioning here that the Hopf and Poisson structures of the $\mathscr{B}$ algebra $Z_{0}$ (defined by generators and relations in $\S 0.5$ ) can be easily calculated using that $\Delta$ is a Poisson map and that the Poisson structure is $\mathscr{B}$-invariant. For example in the case when $\left(a_{i j}\right)$ is symmetric, it suffices to use the following formulas (where $\left.x_{i}=T_{i} y_{i}\right)$ :

$$
\begin{aligned}
& \Delta y_{i}=y_{i} \otimes z_{i}^{-1}+1 \otimes y_{i}, \quad \Delta x_{i}=x_{i} \otimes z_{i}^{-1}+1 \otimes x_{i}, \\
& \Delta z_{\alpha}=z_{\alpha} \otimes z_{\alpha} ; \\
& \left\{z_{\alpha}, z_{\beta}\right\}=0, \quad\left\{y_{i}, z_{\alpha}\right\}=\frac{1}{2}\left(\alpha \mid \alpha_{i}\right) y_{i} z_{\alpha}, \\
& \left\{y_{i}, y_{j}\right\}=0 \text { if } a_{i j}=0, \\
& \left\{y_{i}, y_{j}\right\}=\frac{1}{2} y_{i} y_{j}-T_{i} y_{j} \text { if } a_{i j}=-1, \\
& \left\{z_{i} x_{i}, y_{j}\right\}=\delta_{i j}\left(z_{i}-z_{i}^{-1}\right) .
\end{aligned}
$$

0.11 . We would like to thank the referee whose severe criticism considerably improved the exposition.

\section{The LONGEST Element of the Weyl group $W$}

1.1. Recall that $W$ is a Coxeter group on generators $s_{i}(i=1, \ldots, n)$ and defining relations 


$$
s_{i}^{2}=1 \quad \text { and } \quad\left(s_{i} s_{j}\right)^{m_{i j}}=1 \quad \text { when } i \neq j,
$$

where $m_{i j}=2,3,4$, or 6 for $a_{i j} a_{j i}=0,1,2$, or 3 respectively $(i \neq j)$.

Recall that any element of $W$ has a length $l(w)$ that can be defined as the length of a shortest expression of $w$ as a product of $s_{i}$ and equals the cardinality of $R_{w}:=\left\{\beta \in R^{+} \mid w(\beta)<0\right\}$; such an expression is called a reduced expression of $w$. Recall that

$$
l\left(w s_{i}\right)=l(w)+1 \text { if } w\left(\alpha_{i}\right)>0 \text { and } l\left(w s_{i}\right)=l(w)-1 \text { if } w\left(\alpha_{i}\right)<0 .
$$

1.2. Since $W$ acts transitively on the bases there exists a unique element $w_{0}$ of longest length $N$ such that $w_{0}\left(R^{+}\right)=-R^{+}$. Of course $w_{0}=w_{0}^{-1}$ and $\Pi=-w_{0}(\Pi)$. Let us denote by $j \mapsto \bar{j}$ the permutation of $1,2, \ldots, n$ such that $\alpha_{\bar{j}}=-w_{0}\left(\alpha_{j}\right)$.

We have that $s_{j} w_{0}=w_{0} s_{\bar{j}}$. More precisely, writing $w_{0}=s_{j} s_{i_{1}} s_{i_{2}} \cdots s_{i_{N-1}}=$ $s_{i_{1}} s_{i_{2}} \cdots s_{i_{N-1}} s_{\bar{j}}$ we deduce

Lemma. $s_{i_{1}} s_{i_{2}} \cdots s_{i_{N-1}}\left(\alpha_{\bar{j}}\right)=\alpha_{j}$.

Proof. $s_{i_{1}} s_{i_{2}} \cdots s_{i_{N-1}}\left(\alpha_{\bar{j}}\right)=s_{j} w_{0}\left(\alpha_{\bar{j}}\right)=s_{j}\left(-\alpha_{j}\right)$.

If $w=a b \in W$ is such that $l(w)=l(a)+l(b)$ we will say that this a reduced decomposition.

Given an element $w \in W$ we set $\bar{w}:=w_{0} w w_{0}^{-1}$ (so that $\bar{s}_{j}=s_{\bar{j}}$ ).

1.3. If $w$ is not the longest element there must exist a simple root $\alpha_{i}$ such that $w\left(\alpha_{i}\right)>0$; thus any reduced expression $s_{i_{1}} s_{i_{2}} \cdots s_{i_{m}}$ can be completed to a reduced expression $s_{i_{1}} s_{i_{2}} \cdots s_{i_{N}}$ of $w_{0}$.

In particular given an element $a \in W$ there exist $b, a^{\prime} \in W$ such that $w_{0}=a b=b a^{\prime}$ are reduced decompositions. In this case we clearly have

Lemma. $a^{\prime}=\bar{a}$.

\section{THE BRAID GROUP $\mathscr{B}$}

2.1. Recall that the braid group is an infinite group $\mathscr{B}$ on generators $T_{i}, i=$ $1, \ldots, n$, and the braid relations: for $i \neq j$ we take the word of (even) length $\left(T_{i} T_{j}\right)^{m_{i j}}$, split it in half and impose that the first half be equal to the second written in reverse order. Of course the Weyl group $W$ is the quotient of $\mathscr{B}$ under the further relations $T_{i}^{2}=1$.

It will be convenient to use the abbreviated notation,

$$
T_{i j}^{(m)}=T_{i} T_{j} T_{i} \cdots \quad(m \text { factors }) .
$$

For example, the braid relations read: $T_{i j}^{\left(m_{i j}\right)}=T_{j i}^{\left(m_{j i}\right)}$ if $i \neq j$.

2.2. The main tool of the computations to follow is a result of Matsumoto and Steinberg. 
Theorem. If $s_{i_{1}} s_{i_{2}} \cdots s_{i_{m}}$ is a reduced expression of an element $w \in W$, then the element $T_{w}:=T_{i_{1}} T_{i_{2}} \cdots T_{i_{m}}$ in $\mathscr{B}$ depends only on $w$ (and not on its reduced expression). Moreover, the different reduced expressions of $w$ can be transformed into one another by the braid relations.

This means that we define a canonical section $w \rightarrow T_{w}$ of $W$ in $\mathscr{B}$; of course, this section is not multiplicative but $T_{a} T_{b}=T_{a b}$ if $l(a b)=l(a)+l(b)$. Going back to the longest element we set $T_{0}^{a}:=T_{w_{0}}$ and will often use the following

Lemma. If $a \in W$ we have $T_{a} T_{0}=T_{0} T_{\bar{a}}$.

Proof. From $\S 1.3$ there exists $b$ such that $w_{0}=a b=b \bar{a}$ are reduced decompositions. We thus have $T_{0}=T_{a} T_{b}=T_{b} T_{\bar{a}}$. So $T_{a} T_{0}=T_{a} T_{b} T_{\bar{a}}=T_{0} T_{\bar{a}}$.

Recall another well-known useful fact.

Proposition. If $w_{0}=s_{i_{1}} s_{i_{2}} \cdots s_{i_{N}}$ is a reduced expression of $w_{0}$, then one gets the following ordering of the set of positive roots:

$$
R^{+}=\left\{\alpha_{i_{1}}, s_{i_{1}}\left(\alpha_{i_{2}}\right), s_{i_{1}} s_{i_{2}}\left(\alpha_{i_{3}}\right), \ldots, s_{i_{1}} \cdots s_{i_{N-1}}\left(\alpha_{i_{N}}\right)\right\}
$$

2.3. One of the difficulties in working with quantum groups occurs due to the existence of nontrivial $w \in W$ such that $w\left(\alpha_{i}\right)=\alpha_{h}$. To deal with this difficulty, we need a fact on root systems. Consider a simple root $\alpha_{h}$ and the set $A_{h}$ formed by all the pairs $(w, i)$ such that $w \in W$ and $w\left(\alpha_{i}\right)=\alpha_{h}$. We make this set into a graph by joining $(w, i)$ with $\left(w s_{j}, i\right)$ if $m_{i j}=2$, with $\left(w s_{j} s_{i}, j\right)$ if $m_{i j}=3$, with $\left(w s_{j} s_{i} s_{j}, i\right)$ if $m_{i j}=4$, and with $\left(w s_{j} s_{i} s_{j} s_{i} s_{j}, i\right)$ if $m_{i j}=6$. We have

Lemma. The graph $A_{h}$ is connected.

Proof. We connect any given element $(w, i)$ with $(1, h)$ as follows. Fix a reduced expression of $w$. Then, by $\S 1.1, w s_{i}$ is also a reduced expression and by $\S 1.3$ we can complete it to a reduced expression $w_{0}=w s_{i} w_{1}$. By Proposition 2.2 we have: $R^{+}=\left\{\ldots, w\left(\alpha_{i}\right)=\alpha_{h}, \ldots\right\}$. On the other hand, taking a reduced expression of $w_{0}$ that starts with $s_{h}$, we get an ordering of $R^{+}$that starts with $\alpha_{h}$. Now we pass from the first reduced expression to the second one using braid relations. Then at each step either the braid relation lies entirely in $w$ or $w_{1}$ and then the presentation of $\alpha_{h}$ does not change, or $\alpha_{h}$ becomes an element of the form $\alpha_{h}=w^{\prime}\left(\alpha_{j}\right)$ where $\left(w^{\prime}, j\right)$ is joined with $(w, i)$ in $A_{h}$. At the last step the presentation of $\alpha_{h}$ becomes $\alpha_{h}=1\left(\alpha_{h}\right)$.

Theorem. Let $\mathscr{B}$ act on a set $\mathscr{S}$, and let $u_{1}, \ldots, u_{n} \in \mathscr{S}$ be elements with the properties:

$$
T_{i j}^{(2)} u_{i}=u_{j} \text { if } m_{i j}=3 \text { and } T_{i j}^{\left(m_{i j}-1\right)} u_{j}=u_{j} \text { if } m_{i j}=2,4 \text {, or } 6 \text {. }
$$

Then

(a) For a pair $(w, i)$ in $A_{h}$ we have $T_{w}\left(u_{i}\right)=u_{h}$.

(b) $T_{i}\left(u_{i}\right)=T_{0}\left(u_{\bar{i}}\right)$ and $T_{0}^{2}\left(u_{i}\right)=T_{i}^{2}\left(u_{i}\right)$. 
Proof. (a) is just a restatement of the lemma using the relations of the theorem. As for (b) we have $T_{0}=T_{i} T_{w}=T_{w} T_{\bar{i}}$ where $w=s_{i} w_{0}$ and so $w\left(\alpha_{\bar{i}}\right)=$ $\alpha_{i}$. Thus $T_{i}\left(u_{i}\right)=T_{i} T_{w}\left(u_{\bar{i}}\right)=T_{0}\left(u_{\bar{i}}\right)$, so $T_{i}^{2}\left(u_{i}\right)=T_{i} T_{0}\left(u_{\bar{i}}\right)=T_{0} T_{\bar{i}}\left(u_{\bar{i}}\right)=$ $T_{0}^{2}\left(u_{i}\right)$.

In particular we can apply the theorem to the action of the braid group on a quantum group and the elements $u_{i}=E_{i}$ or $u_{i}=F_{i}$. In this case the relations of the theorem are easily verified (see [L, 5.1]). Thus, we have: if $\alpha_{i}, \alpha_{j}$ are two simple roots and $w \in W$ is such that $w\left(\alpha_{i}\right)=\alpha_{j}$ then $T_{w}\left(E_{i}\right)=E_{j}$. We also have

$$
T_{0}\left(E_{\bar{i}}\right)=T_{i}\left(E_{i}\right)=-F_{i} K_{i}, \quad T_{0}\left(F_{\bar{i}}\right)=T_{i}\left(F_{i}\right)=-K_{i}^{-1} E_{i} .
$$

\section{A UNIVERSAL CONSTRUCTION ASSOCIATED TO THE BRAID GROUP}

3.1. Let $U_{M, \varepsilon}$ be a quantum group at a primitive $l$ th root of unity $\varepsilon, l$ odd. In order to explain our next general construction let us introduce some notations and prove some identities in $U_{M, \varepsilon}$. Let $c_{i}:=\left(\varepsilon^{d_{i}}-\varepsilon^{-d_{i}}\right)^{l}$ and consider the following central elements of $U_{M, \varepsilon}$ :

$$
\begin{gathered}
z_{\beta}:=K_{\beta}^{l} \quad(\beta \in M), \\
z_{i}=z_{\alpha_{i}}, \quad y_{i}:=c_{i} F_{i}^{l}, \quad x_{i}:=T_{0}\left(y_{\bar{i}}\right) \quad(i=1, \ldots, n)
\end{gathered}
$$

(one should remark on the difference in notation used in [DCK]).

By Theorem 2.3 we have $T_{i}\left(y_{i}\right)=T_{0}\left(y_{\bar{i}}\right)$, which implies

(0) $x_{i}=-c_{i} E_{i}^{l} z_{i}^{-1}$.

The following formulas are now immediate by [DCK, (3.3.3 and 4)]:

(1) $T_{i}\left(x_{i}\right)=z_{i}^{2} y_{i}, T_{i}\left(y_{i}\right)=x_{i}, T_{i}\left(z_{\beta}\right)=z_{s_{i} \beta}$.

In order to write down further relations, we introduce one more notation:

$$
y_{i j}^{(m)}=T_{i j}^{(m)} y_{j} \text { if } m \text { is odd and } y_{i j}^{(m)}=T_{i j}^{(m)} y_{i} \text { if } m \text { is even. }
$$

We have the following formulas $(i \neq j)$ :

(2) $y_{i j}^{\left(m_{i j}-1\right)}=y_{j}$.

(3) $y_{i j}^{\left(m_{i j}-2\right)}+y_{j i}^{(1)}=y_{i} y_{j}$ if $m_{i j} \geq 3$ and $a_{i j}=-1$.

(4) $y_{i j}^{\left(m_{i j} / 2-1\right)}-y_{j i}^{\left(m_{i j} / 2\right)}=y_{i}^{2} y_{j}-2 y_{j i}^{(1)} y_{i}$ if $m_{i j} \geq 4$ and $a_{j i}=-1$.

(5) $y_{i j}^{(1)}+y_{j i}^{(4)}=y_{i}^{3} y_{j}-3 y_{i}^{2} y_{j i}^{(1)}+3 y_{i} y_{j i}^{(3)}, y_{i j}^{(3)}-y_{j i}^{(2)}=y_{i}^{3} y_{j}^{2}-3 y_{i}^{2} y_{j} y_{j i}^{(1)}+$ $3 y_{i} y_{j i}^{(1) 2}-3 y_{j i}^{(1)} y_{j i}^{(3)}+y_{j} y_{j i}^{(3)}$ if $m_{i j}=6$ and $a_{j i}=-1$.

Formula (2) follows from 2.3. The remaining formulas are deduced from [L, 5.3-5.5; DCK, (3.4.14)], $§ 5.1$, and the identity

$$
\sum_{j=1}^{l-1}(-1)^{j} \frac{\varepsilon^{-j}}{[j] ![l-j] !}=\frac{c}{2}\left(1-l^{-1}\right), \quad \text { where } c=\left(\varepsilon-\varepsilon^{-1}\right)^{l}
$$


(which follows easily from the Gauss binomial formula: see e.g., [DCK, (1.1.1)]).

Recall that we also have an involution $\omega$, defined by $\omega E_{i}=F_{i}, \omega K_{\beta}=$ $K_{-\beta}$. It commutes with the braid group and we have $\omega\left(z_{\beta}\right)=z_{-\beta}$ and $\omega\left(x_{i}\right)=$ $-z_{i} y_{i}$.

In view of the previous identities we start the following construction.

3.2. Denote by $Z_{0}^{0}$ the algebra with basis $z_{\beta}(\beta \in M)$ and multiplication $z_{\alpha} z_{\beta}=z_{\alpha+\beta}$. The Weyl group, and hence the braid group, acts on $Z_{0}^{0}$ by $s_{i}\left(z_{\beta}\right)=z_{s_{i} \beta}$. Consider the polynomial algebra over $Z_{0}^{0}$ in the indeterminates $T y_{i}$, as $i=1,2, \ldots, n$ and $T \in \mathscr{B}$; we write $y_{i}:=1 y_{i}$. We extend the action of $\mathscr{B}$ from $Z_{0}^{0}$ to this polynomial algebra by

$$
T_{1}\left(T_{2} y_{i}\right):=\left(T_{1} T_{2}\right) y_{i}
$$

We define a quotient algebra of this polynomial algebra by the smallest ideal of relations, stable under $\mathscr{B}$ and containing the following relations:

(1) $T_{i}^{2} y_{i}=z_{i}^{2} y_{i}$

(2) $y_{i j}^{\left(m_{i j}-1\right)}=y_{j}$ if $i \neq j$,

(3) relations $(3)-(5)$ from $\S 3.1$.

Denote by $Z_{0}$ the resulting algebra. Remark that relations (1) and (3) are not homogeneous, and we can also consider the associated homogeneous relations:

(1') $T_{i}^{2}\left(y_{i}\right)=y_{i}$,

$\left(3^{\prime}\right)$ the left-hand sides of (3)-(5) are zero.

In this case we can ignore the variables $z_{i}$ and consider the algebra $D$ over $\mathbb{C}$ generated by the $T y_{i}(T \in \mathscr{B}, i=1, \ldots, n)$ and the relations $\left(1^{\prime}\right),(2)$, and $\left(3^{\prime}\right)$.

3.3. Next given an element $w \in W$ we need to consider the set of all possible reduced expressions $J$ of $w$. If $J: w=s_{i_{1}} s_{i_{2}} \cdots s_{i_{t}}$ is such a reduced expression we define $J^{-1}: w^{-1}=s_{i_{t}} s_{i_{t-1}} \cdots s_{i_{1}}$ a reduced expression of $w^{-1}$. For $k=1, \ldots, t$ we also set

$$
w_{k}^{J}:=s_{i_{1}} s_{i_{2}} \cdots s_{i_{k}}, \quad \beta_{k}^{J}:=s_{i_{1}} s_{i_{2}} \cdots s_{i_{k-1}}\left(\alpha_{i_{k}}\right)=w_{k-1}^{J}\left(\alpha_{i_{k}}\right) .
$$

By Proposition 2.2, the elements $\beta_{k}^{J}$ are distinct positive roots. In fact we have $w^{-1}\left(\beta_{k}^{J}\right)=s_{i_{t}} s_{i_{t-1}} \cdots s_{i_{1}} s_{i_{1}} s_{i_{2}} \cdots s_{i_{k-1}}\left(\alpha_{i_{k}}\right)=-\beta_{t-k+1}^{J^{-1}}$. Thus we get a total ordering of the set $R_{w^{-1}}$.

We can apply this analysis to the element $w_{0}$ and the set of all positive roots. In this case for a given reduced expression $J$ we can construct another reduced expression $\bar{J}: w_{0}=s_{\bar{i}_{1}} s_{\bar{i}_{2}} \cdots s_{\bar{i}_{N}}$. It is clear that $\beta_{k}^{\bar{J}}=-w_{0}\left(\beta_{k}^{J}\right)$.

We now define in $\mathscr{B}$ and in $Z_{0}$ the elements

$$
T_{k-1}^{J}:=T_{i_{1}} T_{i_{2}} \cdots T_{i_{k-1}}, \quad y_{k}^{J}:=T_{i_{1}} T_{i_{2}} \cdots T_{i_{k-1}}\left(y_{i_{k}}\right)=T_{k-1}^{J}\left(y_{i_{k}}\right) .
$$


Let us choose a reduced expression $J: w=s_{i_{1}} s_{i_{2}} \cdots s_{i_{t}}$ of an element $w$. Denote by $Z^{J}$ the $Z_{0}^{0}$-subalgebra of $Z_{0}$ generated by the elements $y_{k}^{J}$, and let $\bar{Z}^{J}$ be its augmentation ideal.

Proposition. One has: $y_{k}^{J}= \pm y_{k}^{J^{\prime}} \bmod \left(\bar{Z}^{J}\right)^{2}$. In particular, $Z^{J}$ and $\bar{Z}^{J}$ are independent of the choice of the reduced expression $J$ of $w$.

Proof. Since one can pass from one expression to another by the use of braid relations we may by induction restrict to the case where $J^{\prime}$ is obtained from $J$ by a single braid relation. We give a proof in the cases $m_{i j}=2$ and $m_{i j}=3$. (In the remaining cases the proof is similar.)

(1) $w=a s_{i} s_{j} b=a s_{j} s_{i} b ; s_{i} s_{j}=s_{j} s_{i} \quad\left(m_{i j}=2\right)$,

(2) $w=a s_{i} s_{j} s_{i} b=a s_{j} s_{i} s_{j} b ; s_{i} s_{j} s_{i}=s_{j} s_{i} s_{j} \quad\left(m_{i j}=3\right)$.

Let $k-1=l(a)$. In case (1) we remark that $y_{h}^{J}=y_{h}^{J^{\prime}}$ if $h \neq k, k+1$, $y_{k}^{J}=T_{a}\left(y_{i}\right)=T_{a} T_{j}\left(y_{i}\right)=y_{k+1}^{J^{\prime}}$, and similarly $y_{k+1}^{J}=y_{k}^{J^{\prime}}$. In case (2) we get $y_{h}^{J}=y_{h}^{J^{\prime}}$ if $h \neq k, k+1, k+2, y_{k}^{J}=T_{a}\left(y_{i}\right)=T_{a} T_{j} T_{i}\left(y_{j}\right)=y_{k+2}^{J^{\prime}}$, and similarly $y_{k}^{J^{\prime}}=y_{k+2}^{J} ; y_{k+1}^{J}=T_{a} T_{i}\left(y_{j}\right)=T_{a}\left(-T_{j}\left(y_{i}\right)-y_{i} y_{j}\right)=-y_{k+1}^{J^{\prime}}-y_{k}^{J} y_{k}^{J^{\prime}}=$ $-y_{k+1}^{J^{\prime}}-y_{k+2}^{J^{\prime}} y_{k}^{J^{\prime}}$.

3.4. The previous proof and Theorem 2.3 have an important

Corollary. (a) If for a given $J$ and $k, \beta_{k}^{J}=\alpha_{i}$ is a simple root then $y_{k}^{J}=y_{i}$.

(b) $T_{i}\left(x_{i}\right)=z_{i}^{2} y_{i}$.

(c) If $\beta_{k}^{J}=\beta_{h}^{J^{\prime}}$ then $y_{k}^{J}= \pm y_{h}^{J^{\prime}}+P$ where $P$ is a polynomial in the elements $y_{i}^{J^{\prime}}$ involving only the indices $i$ for which $\beta_{i}^{J^{\prime}}$ has height strictly less than that of $\beta_{h}^{J^{\prime}}$.

Proof. (a) This is a special case of Theorem 2.3(a).

(b) This follows directly from Theorem 2.3 and the defining relations 3.2.

(c) This follows from the proof of Proposition 3.3.

Remark. The construction and statements of $\S \S 3.2-3.4$, as well as the first statement of Theorem 3.5 below, hold over $\mathbb{Z}$.

3.5. We will now denote $Z_{w}^{-}=Z^{J}$ and $Z_{w}^{+}:=T_{0}\left(Z_{w}^{-}\right)$, and let $x_{k}^{\bar{J}}:=T_{0}\left(y_{k}^{J}\right)$. If $w=w_{0}$ is the longest element we will simply write $Z_{0}^{-}, Z_{0}^{+}$.

Theorem. The algebra $Z_{0}$ is the tensor product $Z_{0}^{-} \otimes Z_{0}^{0} \otimes Z_{0}^{+}$and, given a reduced expression $J$ of $w_{0}$, it is the polynomial ring

$$
Z_{0}^{0}\left[y_{k}^{J}, x_{k}^{J} ; \quad k=1, \ldots, N\right] .
$$

Thus this algebra coincides with the subalgebra $Z_{0}$ of the center of the quantum group $U_{M, \varepsilon}$ studied in [DCK].

Proof. First of all we will show that the subring $S:=Z_{0}^{0}\left[y_{k}^{J}, x_{k}^{J} ; k=1, \ldots, N\right]$ is stable under the action of $\mathscr{B}$, which will prove that it is the entire algebra; 
then we will see that the $2 N$ given elements are algebraically independent by using the quantum groups. Let us begin with the first claim. We need to show that, for a given $j, T_{j}(S) \subset S$.

From the independence of $S$ from $J$ we can choose a reduced decomposition $J$ of $w_{0}$ so that $s_{j}$ appears on the extreme left. Then we have $T_{j}\left(y_{k}^{J}\right)=y_{k+1}^{J}$ unless $k=N$; in the latter case $T_{j}\left(y_{N}^{J}\right)=T_{0}\left(y_{i_{N}}\right)=x_{\bar{i}_{N}}$. Now $T_{j}\left(x_{k}^{J}\right)=T_{j} T_{0}\left(y_{k}^{J}\right)=T_{0} T_{\bar{j}}\left(y_{k}^{J}\right)$. Again $T_{\bar{j}}\left(y_{k}^{J}\right)$ is in $Z_{0}^{-}$and so $T_{j}\left(x_{k}^{J}\right)$ is in $Z_{0}^{+}$unless $y_{k}^{J}=y_{\bar{j}}$, in which case we use 3.2(1) and get $T_{j}\left(x_{\bar{j}}\right)=z_{j}^{2} y_{j}$ as desired.

Next we have to verify the algebraic independence. This follows from the fact that the same statement is true for the subalgebra $Z_{0}$ of the center of the quantum group, and this is a consequence of the existence and the form of a PBW basis (cf. [DCK]).

\section{THE BIG CELL}

4.1. In this section we want to make the link between our formal constructions and the actual Lie algebras and Lie groups. Fix once and for all a sublattice $M$ of $P$ containing $Q$.

Consider the simple Lie algebra $\mathfrak{g}$ corresponding to the Cartan matrix $\left(a_{i j}\right)$, let $\mathfrak{h}$ be a Cartan subalgebra, and let $\mathfrak{g}=\mathfrak{h} \oplus\left(\bigoplus_{\alpha \in R} \mathfrak{g}_{\alpha}\right)$ be the root space decomposition. We denote by $e_{i}, f_{i}, h_{i}$ the usual Chevalley generators. Let $G$ be the corresponding connected algebraic group, and let $T$ be the maximal complex torus of $G$ corresponding to $\mathfrak{h}$ whose group of characters is the lattice $M$. Let $U_{+}$and $U_{-}$denote the unipotent subgroups of $G$ corresponding to positive and negative roots, and let $G^{0}=U_{-} T U_{+}$be the big cell of $G$ (this is a Zariski open dense subset of $G$ ). We set

$$
t_{i}:=\exp \left(f_{i}\right) \exp \left(-e_{i}\right) \exp \left(f_{i}\right) \in G
$$

One knows the following facts that go back to Tits (see, e.g., [KP]):

(1) The mapping $T_{i} \mapsto t_{i}$ extends to a homomorphism of $\mathscr{B}$ to $G$, hence an action of $\mathscr{B}$ on $\mathfrak{g}$.

(2) If $J$ is a reduced expression, setting $f_{k}^{J}=T_{k-1}^{J}\left(f_{i_{k}}\right)$, we have that $f_{k}^{J}$ is a root vector relative to the negative root $-\beta_{k}^{J}$.

4.2. Let us now consider the action of $\mathscr{B}$ on $\mathfrak{g}$. We verify directly the following relations:

(1') $T_{i}^{2}\left(f_{i}\right)=f_{i}$

(2') $T_{i j}^{\left(m_{i j}-1\right)}\left(f_{j}\right)=f_{j}$ if $m_{i j}=2,4$, or 6 .

(3') $T_{j} T_{i}\left(f_{j}\right)=f_{i}, T_{i}\left(f_{j}\right)+T_{j}\left(f_{i}\right)=0$ if $m_{i j}=3$.

(4') $T_{i}\left(f_{j}\right)=T_{j} T_{i}\left(f_{j}\right)$ if $m_{i j}=4$ and $a_{j i}=-1$.

(5') $T_{i j}^{\left(m_{i j}-2\right)}\left(f_{i}\right)+T_{j}\left(f_{i}\right)=0$ if $m_{i j} \geq 4$ and $/ a_{j i}=-1$.

(6) $T_{i j}^{(2)}\left(f_{i}\right)=T_{j i}^{(3)}\left(f_{i}\right), T_{i}\left(f_{j}\right)+T_{j i}^{(4)}\left(f_{j}\right)=0, T_{i j}^{(3)}\left(f_{j}\right)=T_{j i}^{(2)}\left(f_{j}\right)$ if $m_{i j}=6$ and $a_{j i}=1$. 
We can take these relations to define a commutative algebra $D$ with a $\mathscr{B}$-action.

Proposition. The subalgebra generated in $S(\mathfrak{g})$ by the $\mathscr{B}$-translates of the $f_{i}$ is $\mathscr{B}$-isomorphic to the algebra $D$.

Proof. The $\mathscr{B}$-equivariant mapping sending $y_{i}$ to $f_{i}$ is well defined since the relations defining $D$ hold in our ring. $D$ is generated by the elements $y_{k}^{J}$ and $x_{k}^{J}$ that map to the corresponding vectors $f_{k}^{J}$ and $e_{k}^{J}$. These vectors are linearly independent (in $\mathfrak{g}$ ) and this finishes the proof.

4.3. We return now to the algebra $Z_{0}=Z_{0}^{-} \otimes Z_{0}^{0} \otimes Z_{0}^{+}$, and let $\Omega_{M}=\operatorname{Spec} Z_{0}$ be the algebraic variety of its $\mathbb{C}$-valued points. Thus $\Omega_{M}$ is a product of the $N$-dimensional affine space $\operatorname{Spec} Z_{0}^{-}$, the complex torus $T=\operatorname{Spec} Z_{0}^{0}$ (we identify $Z_{0}^{0}$ with $U_{\varepsilon}^{0}=\mathbb{C}[T]$ via the map $z_{\alpha} \mapsto K_{\alpha}$ ), and the $N$-dimensional affine space $\operatorname{Spec} Z_{0}^{+}$. The group $\mathscr{B}$ acts on $\Omega_{M}$ algebraically. We act with $\mathscr{B}$ on $G$ by inner conjugation by the elements $t_{i}: T_{i}(g):=t_{i} g t_{i}^{-1}$. We consider the set $\mathscr{F}$ of (regular) maps $F: \Omega_{M} \rightarrow G$ and act on $\mathscr{F}$ with $\mathscr{B}$ in the obvious way: $\left(T_{i} F\right)(p):=T_{i}\left(F\left(T_{i}^{-1}(p)\right)\right)$. We construct now some special maps $\Omega_{M} \rightarrow G^{0}$

$$
Y_{k}=\exp \left(y_{k} f_{k}\right): p \mapsto \exp \left(y_{k}(p) f_{k}\right)
$$

for a reduced expression $J$,

$$
Y_{k}^{J}:=\exp \left(y_{k}^{J} f_{k}^{J}\right)
$$

and finally the map $Z$ that is trivial on the first and the third factors and is induced on the second factor by the map $\beta \mapsto 2 \beta, \beta \in M$.

Since $y_{k}^{J}=T_{k-1}^{J}\left(y_{i_{k}}\right)$ and $f_{k}^{J}=t_{k-1}^{J}\left(f_{i_{k}}\right)$ we get from the definition of the braid group action on $\mathscr{F}$ that

$$
Y_{k}^{J}=T_{k-1}^{J}\left(Y_{i_{k}}\right)
$$

Lemma. If $\beta_{k}^{J}=\alpha_{i}$ is a simple root we have $Y_{k}^{J}=Y_{i}$.

Proof. This follows from Corollary 3.4 and the analogous statement for the action of $\mathscr{B}$ on the root vectors.

4.4. Lemma. (1) $Y_{i} T_{i}\left(Y_{j}\right)=Y_{j} T_{j}\left(Y_{i}\right)$ if $m_{i j}=2$.

(2) $Y_{i} T_{j}\left(Y_{i}\right) Y_{j}=Y_{j} T_{i}\left(Y_{j}\right) Y_{i}$ if $m_{i j}=3$.

(3) $Y_{i} T_{j} T_{i}\left(Y_{j}\right) T_{j}\left(Y_{i}\right) Y_{j}=Y_{j} T_{i} T_{j}\left(Y_{i}\right) T_{i}\left(Y_{j}\right) Y_{i}$ if $m_{i j}=4$.

(4) $Y_{i}\left(T_{j i}^{(4)} Y_{j}\right)\left(T_{j i}^{(3)} Y_{i}\right)\left(T_{j i}^{(2)} Y_{j}\right)\left(T_{j} Y_{i}\right) Y_{j}=Y_{j}\left(T_{i j}^{(4)} Y_{i}\right)\left(T_{i j}^{(3)} Y_{j}\right)\left(T_{i j}^{(2)} Y_{i}\right)\left(T_{i} Y_{j}\right) Y_{i}$ if $m_{i j}=6$.

Proof. By direct calculation. (1) is clear and as for (2) we can compute in $\mathrm{SL}_{3}$. With the usual notation of elementary matrices we have

$$
\begin{array}{cl}
Y_{1}=1+y_{1} e_{21}, & Y_{2}=1+y_{2} e_{32}, \\
t_{1}=-e_{12}+e_{21}+e_{33}, & t_{2}=e_{11}-e_{23}+e_{32} .
\end{array}
$$


Hence we get

$$
T_{1}\left(Y_{2}\right)=t_{1}\left(1+T_{1}\left(y_{2}\right) e_{32}\right) t_{1}^{-1}=1-T_{1}\left(y_{2}\right) e_{31}
$$

and

$$
T_{2}\left(Y_{1}\right)=t_{2}\left(1+T_{2}\left(y_{1}\right) e_{21}\right) t_{2}^{-1}=1+T_{2}\left(y_{1}\right) e_{31}
$$

so

while

$$
Y_{1} T_{2}\left(Y_{1}\right) Y_{2}=1+y_{1} e_{21}+T_{2}\left(y_{1}\right) e_{31}+y_{2} e_{32}
$$

$$
Y_{2} T_{1}\left(Y_{2}\right) Y_{1}=1+y_{1} e_{21}-T_{1}\left(y_{2}\right) e_{31}+y_{2} e_{32}+y_{1} y_{2} e_{31}
$$

thus the relation follows from 3.1(3). The proofs of (3) and (4) is similar.

4.5. We define now, for $J: w=s_{i_{1}} s_{i_{2}} \cdots s_{i_{t}}$ the maps

$$
Y^{J}:=Y_{t}^{J} Y_{t-1}^{J} \cdots Y_{1}^{J}, \quad X^{J}:=T_{0}\left(Y^{J}\right) .
$$

Proposition. (1) $Y^{J}$ and $X^{J}$ are independent of $J$; we denote them: $Y_{w}$ and $X_{\bar{w}}$.

(2) If $w=a b$ is a reduced decomposition then

$$
Y_{w}=T_{a}\left(Y_{b}\right) Y_{a} \text { and } X_{w}=T_{a}\left(X_{b}\right) X_{a} .
$$

(3) $Y_{w}$ can be thought of as an algebraic isomorphism between the affine space with coordinates $y_{k}^{J}$ and the unipotent group $w^{-1}\left(U_{+}\right) \cap U_{-}$.

Proof. (1) It suffices to do it for $Y^{J}$. Again it is enough to do it for two reduced expressions which differ by a single braid relation. In this case we see that the factors in the two products coincide except for two or three, etc. consecutive ones. Then Lemmas 4.3 and 4.4 finish the proof.

(2) This is clear from the definitions for the $Y_{w}$ and follows from Lemma 2.2 for the $X_{w}$.

(3) $Y_{w}$ is the product of the root subgroups relative to the roots $-\beta_{k}^{J}$ and these are the negative roots that $w$ maps to positive roots.

We shall refer to $Y$ and $X$ instead of $Y_{w_{0}}$ and $X_{w_{0}}$. We shall also write $X_{i}$ in place of $X_{s_{i}}=\exp \left(-x_{i} e_{i}\right)$.

4.6. Proposition. $T_{i}(Y)=T_{i}\left(Y_{i}\right) Y Y_{i}^{-1}, T_{i}(X)=T_{i}\left(X_{i}\right) X X_{i}^{-1}$, and $T_{i}(Z)=$ $Z$.

Proof. Let $w_{0}=s_{i} w=w s_{\bar{i}}$ so that, from Proposition 4.5(2), we have $Y=$ $T_{w}\left(Y_{\bar{i}}\right) Y_{w}=T_{i}\left(Y_{w}\right) Y_{i}$. But, since $w\left(\alpha_{\bar{i}}\right)=\alpha_{i}$, from Lemma 4.3 we have $T_{w}\left(Y_{\bar{i}}\right)=Y_{i}$ and so

$$
Y=Y_{i} Y_{w}, \quad T_{i}(Y)=T_{i}\left(Y_{i}\right) T_{i}\left(Y_{w}\right)=T_{i}\left(Y_{i}\right) Y Y_{i}^{-1}
$$

Furthermore,

$$
\begin{aligned}
T_{i}(X) & =T_{i} T_{0}(Y)=T_{0} T_{\bar{i}}(Y)=T_{0}\left(T_{\bar{i}}\left(Y_{\bar{i}}\right) Y Y_{\bar{i}}^{-1}\right) \\
& =T_{0}\left(T_{\bar{i}}\left(Y_{\bar{i}}\right)\right) X T_{0}\left(Y_{\bar{i}}^{-1}\right)=T_{i}\left(T_{0}\left(Y_{\bar{i}}\right)\right) X T_{0}\left(Y_{\bar{i}}^{-1}\right)=T_{i}\left(X_{i}\right) X X_{i}^{-1}
\end{aligned}
$$


Finally, the statement for $Z$ is clear from the definitions.

4.7. Theorem. $T_{i}(Y Z X)=X_{i} Y Z X X_{i}^{-1}$.

Proof. From Proposition 4.6 we have $T_{i}(Y Z X)=X_{i} Y Y_{i}^{-1} Z T_{i}\left(X_{i}\right) X X_{i}^{-1}$, so we need to show that $Z=Y_{i}^{-1} Z T_{i}\left(X_{i}\right)$ or $Z^{-1} Y_{i} Z=T_{i}\left(X_{i}\right)$. We write $T_{i}\left(X_{i}\right)=\exp \left(-T_{i}\left(x_{i}\right) t_{i}\left(e_{i}\right)\right)=\exp \left(z_{i}^{2} y_{i} f_{i}\right)$. But by the definition of the map $Z$ we have that $Z^{-1} \exp \left(y_{i} f_{i}\right) Z=\exp \left(z_{i}^{2} y_{i} f_{i}\right)$.

Corollary. The map $Y Z X$ of $\Omega_{M}$ onto the big cell $U_{-} \times T \times U_{+}$is of degree $2^{n}$, and the functions on the group $G$ invariant under conjugation pullback to functions on $\Omega_{M}$ invariant under $\mathscr{B}$.

Proof. The first part is clear from the definitions. If we restrict a $G$-invariant function $f$ from $G$ to the open cell we have

$$
\begin{aligned}
T_{i}(f(Y Z X(p))) & =f\left(Y Z X\left(T_{i}^{-1}(p)\right)\right)=f\left(t_{i}\left(Y Z X\left(T_{i}^{-1}(p)\right)\right) t_{i}^{-1}\right) \\
& =f\left(T_{i}(Y Z X)(p)\right)=f\left(X_{i} Y Z X X_{i}^{-1}(p)\right)=f(Y Z X(p)) .
\end{aligned}
$$

Corollary. For $w \in W$ we have $T_{w}(Y Z X)=X_{w} Y Z X X_{w}^{-1}$.

Proof. This is an immediate consequence of the theorem and Proposition 4.5(2) using induction on the length of $w$.

In particular $T_{0}(Y Z X)=X Y Z$, and since $X=T_{0}(Y)$, we have $T_{0}(X)=$ $Z^{-1} Y Z$.

Remark. One can connect the formulas with the Cartan involution $\sigma$ in $G$ and the transformation $\omega$. In fact $\sigma \omega\left(Y_{k}^{J}\right)=\exp \left(z_{\beta_{k}^{J}} x_{k}^{J} e_{k}^{J}\right)$ so $\sigma \omega(Y)=$ $Z^{1 / 2} X Z^{-1 / 2}$.

\section{THE QUANTUM COADJOINT ACTION}

5.1. As in [DCK, §3.4] suppose one has an element $b \in U_{M, \mathscr{A}}$ with the property that $[b, a] \in[l] U_{M, \mathscr{A}}$ for all $a \in U_{M, \mathscr{A}}$. Then when we set $q=\varepsilon$, we have that $b$ is central in $U_{M, \varepsilon}$ but one can also define the derivation $D_{b}$ of $U_{M, \varepsilon}$ by $D_{b}(a):=[b /[l], a]_{q=\varepsilon}$. In particular for $b=E_{i}^{l}$ we have the derivations $\underline{e}_{i}$ of $U_{M, \varepsilon}$ given by $\underline{e}_{i}(a):=\left[E_{i}^{(l)}, a\right]_{q=\varepsilon}$. The following formulas can be derived from [L, 5.3-5.5; DCK, §3.4] (with our change of notations):

$$
\begin{aligned}
& \underline{e}_{i}\left(z_{\alpha}\right)=\left(\alpha \mid \alpha_{i}\right) z_{\alpha+\alpha_{i}} x_{i} /\left(\alpha_{i} \mid \alpha_{i}\right) ; \\
& \underline{e}_{i}\left(x_{i}\right)=-z_{i} x_{i}^{2} ; \\
& \underline{e}_{i}\left(x_{j}\right)=z_{i} x_{j i}^{\left(m_{j i}-2\right)} \text { if } i \neq j \text { and }\left|a_{i j}\right| \leq\left|a_{j i}\right| ; \\
& \underline{e}_{i}\left(x_{j}\right)=-a_{i j} z_{i} T_{j}\left(x_{i}\right) \text { if } a_{j i}=-1 ; \\
& \underline{e}_{i}\left(T_{j}\left(x_{i}\right)\right)=0 \text { if } m_{i j}=3 ; \\
& \underline{e}_{i}\left(T_{j}\left(x_{i}\right)\right)=z_{i} T_{j} T_{i}\left(x_{j}\right), \underline{e}_{i}\left(T_{j} T_{i}\left(x_{j}\right)\right)=z_{i} T_{j}\left(x_{i}\right) \text { if } m_{i j}=4 \text { and } a_{j i}=-1 ; \\
& \underline{e}_{i}\left(T_{j}\left(x_{i}\right)\right)=z_{i}\left(T_{j} T_{i}\left(x_{j}\right)\right)^{2}, \underline{e}_{i}\left(T_{j} T_{i}\left(x_{j}\right)\right)=0 \text { if } m_{i j}=4 \text { and } a_{i j}=-1 ; \\
& \underline{e}_{i}\left(T_{j}\left(x_{i}\right)\right)=2 z_{i} x_{j i}^{(3)}, \underline{e}_{i}\left(x_{j i}^{(3)}\right)=z_{i} x_{j i}^{(4)} ;
\end{aligned}
$$




$$
\begin{aligned}
& \underline{e}_{i}\left(x_{j i}^{(2)}\right)=3 z_{i} x_{j i}^{(3) 2} \text { if } m_{i j}=6 \text { and } a_{j i}=-1 ; \\
& \underline{e}_{i}\left(x_{j}^{(4)}\right)=0 \text { if } m_{i j}=6 ; \\
& \underline{e}_{i}\left(x_{j i}^{(3)}\right)=z_{i} x_{j i}^{(4) 3}, \underline{e}_{i}\left(x_{j i}^{(2)}\right)=z_{i} x_{j i}^{2} ; \\
& \underline{e}_{i}\left(T_{j}\left(x_{i}\right)\right)=3 z_{i} x_{j i}^{(4)} x_{j i}^{(2)}-z_{i} x_{j i}^{(3)} \text { if } m_{i j}=6 \text { and } a_{i j}=-1 ; \\
& \underline{e}_{i}\left(y_{j}\right)=\delta_{i j}\left(z_{i}-z_{i}^{-1}\right) ; \\
& \underline{e}_{i}\left(T_{i}\left(y_{j}\right)\right)=z_{i} y_{j} \text { if } a_{i j}=-1 ; \\
& \underline{e}_{i}\left(T_{i}\left(y_{j}\right)\right)=-a_{i j} z_{i} T_{i} T_{j}\left(y_{i}\right) \text { if } a_{j i}=-1 ; \\
& \underline{e}_{i}\left(T_{i} T_{j}\left(y_{i}\right)\right)=z_{i} y_{j}^{2} \text { if } m_{i j}=4 \text { and } a_{i j}=-1 ; \\
& \underline{e}_{i}\left(y_{i j}^{(3)}\right)=3 z_{i} y_{i j}^{(4) 2}, \underline{e}_{i}\left(y_{i j}^{(2)}\right)=2 z_{i} y_{i j}^{(4)} ; \\
& \underline{e}_{i}\left(y_{i j}^{(4)}\right)=z_{i} y_{j} \text { if } m_{i j}=6 \text { and } a_{j i}=-1 ; \\
& \underline{e}_{i}\left(y_{i j}^{(3)}\right)=z_{i} y_{j}^{2}, e_{i}\left(y_{i j}^{(4)}\right)=z_{i} y_{j}^{3} ; \\
& \underline{e}_{i}\left(y_{i j}^{(2)}\right)=-z_{i} y_{i j}^{(4)}+3 z_{i} y_{j} y_{i j}^{(3)} \text { if } m_{i j}=6 \text { and } a_{i j}=-1 .
\end{aligned}
$$

Finally, if $\phi$ is any automorphism of $U_{M, \varepsilon}$ we obviously have $\phi D_{b} \phi^{-1}(a)=$ $D_{\phi(b)}(a)$ or in other form (substituting $\phi^{-1}(a)$ for $\left.a\right), \phi\left(D_{b}(a)\right)=D_{\phi(b)}(\phi(a))$. An important case is when $\alpha_{i}, \alpha_{j}$ are two simple roots and $w \in W$ is such that $w\left(\alpha_{i}\right)=\alpha_{j}$ so that $T_{w}\left(E_{i}\right)=E_{j}$. Thus we have, taking $\phi=T_{w}$,

$$
T_{w}\left(\underline{e}_{i}(a)\right)=\underline{e}_{j}\left(T_{w}(a)\right) .
$$

In order to perform our computations we need some preliminary steps.

5.2. For $\mathrm{SL}_{2}$ we have the elements

$$
Y=\left(\begin{array}{ll}
1 & 0 \\
y & 1
\end{array}\right), \quad Z=\left(\begin{array}{cc}
z & 0 \\
0 & z^{-1}
\end{array}\right), \quad X=\left(\begin{array}{cc}
1 & -x \\
0 & 1
\end{array}\right)
$$

and their product

$$
A:=\left(\begin{array}{cc}
z & -z x \\
z y & -z x y+z^{-1}
\end{array}\right) .
$$

We apply the operator $\underline{e}$ to $A$ and get by the formulas in $\S 5.1$,

$$
\underline{e}(A)=\left(\begin{array}{cc}
z^{2} x & 0 \\
z^{2} x y+z^{2}-1 & -z^{2} x
\end{array}\right)=\left[z e_{21}, A\right] .
$$

(We have dropped the subscript 1 in all formulas.)

5.3. Next, we show that for $\mathrm{G}=\mathrm{SL}_{3}$, again if $A=Y Z X$ then

$$
\underline{e}_{1}(A)=\left[z_{1} e_{21}, A\right] \text {. }
$$

We have by Proposition 4.5(2), $Y=\bar{Y} Y_{1}$, where $\bar{Y}=T_{1}\left(Y_{s_{2} s_{1}}\right)$ and $X=$ $T_{s_{2} s_{1}}\left(X_{2}\right) X_{s_{2} s_{1}}=X_{1} \bar{X}$, where $\bar{X}=X_{s_{2} s_{1}}$, since the longest element of $W$ is $s_{1} s_{2} s_{1}=s_{2} s_{1} s_{2}$. We also decompose $Z=Z_{1} \bar{Z}$, where $\bar{Z}$ is the kernel of $\alpha_{1}$ 
in $T$. Explicitly,

$$
\begin{gathered}
Y_{1}=1+y_{1} e_{21}, \quad Y_{2}=1+y_{2} e_{32} ; \\
t_{1}=-e_{12}+e_{21}+e_{33}, \quad t_{2}=e_{11}-e_{23}+e_{32}, \quad t_{0}:=t_{1} t_{2} t_{1}=e_{31}-e_{22}+e_{13} ; \\
Y_{s_{2} s_{1}}=T_{2}\left(Y_{1}\right) Y_{2}=1+y_{2} e_{32}+T_{2}\left(y_{1}\right) e_{31} ; \\
\bar{Y}=1-T_{1}\left(y_{2}\right) e_{31}+y_{2} e_{32} ; \\
Z_{1}=\operatorname{diag}\left(z_{\omega_{1}}^{2} z_{\omega_{2}}^{-2}, z_{\omega_{1}}^{-2}, z_{\omega_{2}}^{2}\right), \quad \bar{Z}=\operatorname{diag}\left(z_{\omega_{2}}^{2}, z_{\omega_{2}}^{2}, z_{\omega_{2}}^{-4}\right) \\
X_{1}=t_{0} Y_{2} t_{0}=1-x_{1} e_{12}, \quad X_{2}=t_{0} Y_{1} t_{0}=1-x_{2} e_{23} \\
\bar{X}=T_{2}\left(X_{1}\right) X_{2}=1-T_{2}\left(x_{1}\right) e_{13}-x_{2} e_{23} .
\end{gathered}
$$

Using $§ 5.1$, we obtain

$$
\begin{aligned}
& \underline{e}_{1}(\bar{Y})=-z_{1} y_{2} e_{31}=\left[z_{1} e_{21}, \bar{Y}\right] ; \\
& \underline{e}_{1}(\bar{X})=-z_{1} T_{2}\left(x_{1}\right) e_{23}=\left[z_{1} e_{21}, \bar{X}\right] \\
& \underline{e}_{1}(\bar{Z})=0=\left[z_{1} e_{21}, \bar{Z}\right] .
\end{aligned}
$$

Finally since $A=\bar{Y}\left(Y_{1} Z_{1} X_{1}\right) \overline{Z X}$ and the derivations $\underline{e}_{1}$ and $z_{1}$ ade $e_{21}$ coincide on all four factors by the above formulas and (a version of) $\S 5.2$, we deduce that they coincide on $A$.

5.4. Consider again the big cell $G^{0}=U_{-} T U_{+}$and the mapping $Y Z X$ that is an unramified covering: $\Omega_{M} \rightarrow G^{0}$. Thus given a vector field on $G$ we can pull it back to $\Omega_{M}$. We will do this with the elements of the Lie algebra $\mathfrak{g}$, in particular with the Chevalley generators $e_{i}, h_{i}$, and $f_{i}$. We consider a representation $\rho$ of $G$ and its Lie algebra $\mathfrak{g}$. In a given basis the entries of the matrix $\rho(g)$ are functions on $G$ and if $a \in \mathfrak{g}$ the entries of $[a, \rho(g)]$ are the derivatives of the entries of $\rho(g)$ according to the vector field $a$. A mapping $f$ of $\Omega_{M}$ in $G$ can be composed with $\rho$ to get a map to matrices. We will drop the symbol $\rho$ if there is no ambiguity. For the pull back $a^{*}$ of $a$ we have of course the same formula $[a, \rho(f(g))]=a^{*}(\rho(f(g)))$. We wish to show that

Theorem. $\underline{e}_{i}=z_{i} f_{i}$.

Proof. We have made the computation for $\mathrm{SL}_{2}$ and $\mathrm{SL}_{3}$. Similar but lengthier calculations for the four-dimensional representation of $C_{2}$ and the sevendimensional representation of $G_{2}$ show that the formula hold for all rank 2 groups as well. We will reduce the general case to these cases.

For each $\alpha \in R^{+}$pick $w_{\alpha} \in W$ such that $\alpha=w_{\alpha}\left(\alpha_{j}\right), \alpha_{j} \in \Pi$, and let

$$
y_{\alpha}=T_{w_{\alpha}} y_{j}, \quad x_{\alpha}=T_{w_{\alpha}} x_{j} .
$$

Since, by Corollary 3.4(c), the elements $x_{\alpha}$ and $y_{\alpha}$ generate $Z_{0}$ over $Z_{0}^{0}$ and since the derivatives $\underline{e}_{i}$ and $z_{i} f_{i}$ coincide on $Z_{0}^{0}$ by the rank 2 calculations, it suffices to show that

$$
\underline{e}_{i}\left(y_{\alpha}\right)=z_{i} f_{i}\left(y_{\alpha}\right), \quad \underline{e}_{i}\left(x_{\alpha}\right)=z_{i} f_{i}\left(x_{\alpha}\right), \quad \alpha \in R^{+} .
$$


Given two nonproportional roots $\alpha$ and $\beta$, we denote by $R_{\alpha, \beta}$ the intersection of the $\mathbb{Z}$-span of $\alpha$ and $\beta$ with $R$ and let $R_{\alpha, \beta}^{+}=R_{\alpha, \beta} \cap R^{+}$. Then $R_{\alpha, \beta}$ is a rank 2 root system with $R_{\alpha, \beta}^{+}$being a subset of positive roots.

There exist two simple roots, say, $\alpha_{1}, \alpha_{2} \in \Pi$, and $w \in W$ such that $w R_{\alpha_{1}, \alpha_{2}}^{+}=R_{\alpha_{i}, \alpha}^{+}$and $w \alpha_{1}=\alpha_{i}\left(\alpha\right.$ need not be equal to $\left.w\left(\alpha_{2}\right)\right)$.

Fix a reduced expression $w=s_{i_{1}} \cdots s_{i_{k}}$. Let $w_{0}^{\prime}=s_{1} s_{2} s_{1} \cdots s_{\varepsilon}$, where $\varepsilon=1$ or 2 , be the reduced expression $J^{\prime}$ of the longest element of the Weyl group of $R_{\alpha_{1}, \alpha_{2}}$, and let $m=l\left(w_{0}^{\prime}\right)$. Then the expression $w w_{0}^{\prime}=s_{i_{1}} \cdots s_{i_{k}} s_{1} s_{2} \cdots s_{\varepsilon}$ is reduced; this is easily checked by using $\S 1.1$. Complete to the right this expression to a reduced expression $J$ of $w_{0}$. Let $\left\{\beta_{1}, \ldots, \beta_{N}\right\}$ be the corresponding ordering of $R^{+}$associated to this reduced expression (see Proposition 2.1). Then $R^{+}$breaks into four pieces:

$$
\begin{aligned}
& R^{1}:=\left\{\beta_{1}, \ldots, \beta_{k}\right\}=R_{w^{-1}}, \quad \beta_{k+1}=\alpha_{i}, \\
& R^{2}:=\left\{\beta_{k+2}, \ldots, \beta_{k+m}\right\}=R_{\alpha_{i}, \alpha}^{+} \backslash\left\{\alpha_{i}\right\}, \quad R^{3}=\left\{\beta_{k+m+1}, \ldots, \beta_{N}\right\} .
\end{aligned}
$$

Furthermore, let $\mathfrak{g}_{ \pm}^{i}=\bigoplus_{\gamma \in R^{i}} \mathbb{C} e_{ \pm \gamma}, \quad i=1,2,3$. It is easy to see that these are subalgebras of the Lie algebra $\mathfrak{g}$ normalized by the three-dimensional subalgebra $\mathbb{C} e_{i}+\mathbb{C} h_{i}+\mathbb{C} f_{i}$. Indeed, we have to show that for $s=1$ and 3 , the $R^{s}$ are additively closed subsets of $R^{+}$such that $R \cap\left(R^{s} \pm \alpha_{i}\right) \subset R^{s}$. Since $R^{1}=R_{w^{-1}}$, it is additively closed. If $\beta \in R^{1}$ and $\beta \pm \alpha_{i} \in R$, then $\beta \pm \alpha_{i} \in R^{+}$ since $\alpha_{i}$ is simple and $w^{-1}\left(\beta \pm \alpha_{i}\right)=w^{-1}(\beta) \pm \alpha_{1} \in-R^{+}$since $\alpha_{1}$ is simple. Furthermore, $R^{3}=R^{+} \backslash R_{w_{0}^{\prime} w^{-1}}$ and hence is additively closed. If $\beta \in R^{3}$ and $\beta \pm \alpha_{i} \in R$, then, as above, $\beta \pm \alpha_{i} \in R^{+}$and $w^{-1}\left(\beta \pm \alpha_{i}\right)=w^{-1}(\beta) \pm \alpha_{1} \in R^{+}$; hence $w_{0}^{\prime} w^{-1}\left(\beta \pm \alpha_{i}\right)=w_{0}^{\prime} w^{-1}(\beta) \pm w_{0}^{\prime}\left(\alpha_{1}\right) \in R^{+}$since $-w_{0}^{\prime}\left(\alpha_{1}\right)$ is simple. Let $U_{ \pm}^{i}$ be the subgroups of $U_{ \pm}$corresponding to the $\mathfrak{g}_{ \pm}^{i}$.

We turn now to the map $Y Z X$. We have the decompositions of $Y$ and $X$ according to the above decomposition of $R^{+}$

$$
\begin{aligned}
Y Z X & =Y_{3} Y_{2}\left(\exp y_{i} f_{i}\right) Y_{1} Z X_{1}\left(\exp -x_{i} e_{i}\right) X_{2} X_{3} \\
& =Y_{3} Y_{2} Y_{1}^{\prime}\left(\left(\exp y_{i} f_{i}\right) Z\left(\exp -x_{i} e_{i}\right)\right) X_{1}^{\prime} X_{2} X_{3},
\end{aligned}
$$

where $Y_{1}^{\prime}=\left(\exp y_{i} f_{i}\right) Y_{1}\left(\exp -y_{i} f_{i}\right) \subset U_{-}^{1}$ and $X_{1}^{\prime}=\left(\exp -x_{i} e_{i}\right) X_{1}\left(\exp x_{i} e_{i}\right) \epsilon$ $U_{+}^{1}$.

Consider the subalgebra $Z_{0}^{1,2}$ of $Z_{0}$ generated over $Z_{0}^{0}$ by all $x_{\gamma}$ and $y_{\gamma}$ with $\gamma \in R_{\alpha_{1}, \alpha_{2}}^{+}$. We want to prove

$$
\left(z_{i} f_{i}\right)\left(T_{w}(a)\right)=T_{w}\left(z_{1} f_{1}(a)\right) \text { for } a \in Z_{0}^{1,2} \text {. }
$$

This formula implies (1). Indeed using the formula at the end of $\S 5.1$ and the calculation in the rank 2 case, we have for $a \in Z_{0}^{1,2}, \underline{e}_{i}\left(T_{w}(a)\right)=T_{w} \underline{e}_{1}(a)=$ $T_{w}\left(z_{1} f_{1}(a)\right)$. 
In order to prove (2) note that the action of $z_{i} f_{i}$ on $Z_{0}$ may be calculated as follows. Write for $t \in \mathbb{C}$

$$
\left(\exp t z_{i} f_{i}\right) Y Z X\left(\exp -t z_{i} f_{i}\right)=\prod_{s=N}^{1}\left(\exp y_{s}^{J}(t) f_{s}^{J}\right) Z(t) \prod_{s=1}^{N}\left(\exp x_{s}^{J}(t) e_{s}^{J}\right) .
$$

Then $z_{i} f_{i}\left(x_{s}^{J}\right)=\left.\frac{d}{d t} x_{s}^{J}(t)\right|_{t=0}$, and similarly for $y$.

But $x_{\alpha}$ (resp. $y_{\alpha}$ ) occurs only in $X_{2}$ (resp. $Y_{2}$ ) and all other factors of $Y Z X$ lie in the subgroups normalized by $\exp t z_{i} f_{i}$ and having trivial intersection with $U_{+}^{2}\left(\right.$ resp. $\left.U_{-}^{2}\right)$. Thus, it suffices to perform the calculation in $U_{+}^{2}$ (resp. $\left.U_{-}^{2}\right)$. We have

$$
\begin{aligned}
\prod_{s=k+2}^{k+m} \exp x_{s}^{J}(t) e_{s}^{J} & =\left(\exp t z_{i} f_{i}\right) \prod_{s=2}^{m} \exp T_{w}\left(x_{s}^{J^{\prime}} e_{s}^{J^{\prime}}\right)\left(\exp -t z_{i} f_{i}\right) \\
& =T_{w}\left(\left(\exp t z_{1} f_{1}\right)\left(\prod_{s=2}^{m} \exp x_{s}^{J^{\prime}} e_{s}^{J^{\prime}}\right)\left(\exp -t z_{1} f_{1}\right)\right),
\end{aligned}
$$

and we can use again the calculation in the rank 2 case. This proves (2).

In the next section we shall need the following result.

Lemma. Let $\beta \in R^{+}$and let $p \in \mathbb{Z}_{+}$be such that $\beta+p \alpha_{i} \in R^{+}$but $\beta+$ $(p+1) \alpha_{i} \notin R^{+}$. Then for suitable choices of $x_{\beta}$ and $x_{\beta+\alpha_{i}}$ one has

$$
\underline{e}_{i}\left(x_{\beta}\right)=p z_{i} x_{\beta+\alpha_{i}} .
$$

Proof. If $\beta \in R_{\alpha_{1}, \alpha_{2}}^{+}$for some simple roots $\alpha_{1}$ and $\alpha_{2}$, and $i=1$, the lemma holds due to $\S 5.1$. This case implies the general one if we take $w \in W$ such that $w R_{\alpha_{1}, \alpha_{2}}^{+}=R_{\alpha_{i}, \beta}^{+}$, and $w \alpha_{1}=\alpha_{i}$, and apply $T_{w}$.

5.5. Let

$$
\underline{f}_{i}=T_{0} \underline{e}_{i} T_{0}^{-1}
$$

As in $\S 2.3$, we have

$$
\underline{f}_{i}=T_{i} \underline{e}_{i} T_{i}^{-1} .
$$

As in $\S 5.4$, it suffices to verify the following formula in the rank 1 and 2 cases where we verify it as in $\S 5.3$.

Theorem. $\underline{f}_{i}=-z_{i} e_{i}$.

Recall that given functions $f, g$ and vector fields $U, V$, one has

$$
[f U, g V]=f g[U, V]+f U(g) V-g V(f) U .
$$

Using this, we immediately deduce the

Lemma. (a) $\underline{h}_{i}:=\left[\underline{e}_{i}, \underline{f}_{i}\right]=z_{i}^{2} h_{i}+z_{i}^{2} x_{i} e_{i}+y_{i} f_{i}$.

(b) $\left[\underline{e}_{i_{1}},\left[\underline{e}_{i_{2}},\left[\ldots,\left[\underline{e}_{i_{k-1}}, \underline{e}_{i_{k}}\right] \ldots\right]=z_{\beta}\left[f_{i_{1}},\left[f_{i_{2}},\left[\ldots,\left[f_{i_{k-1}}, f_{i_{k}}\right] \ldots\right]+f\right.\right.\right.\right.$, where $\beta=\alpha_{i_{1}}+\cdots+\alpha_{i_{k}}$ and $f$ is a linear combination of vector fields (with 
coefficients functions) corresponding to root vectors attached to roots $-\alpha$ such that $\alpha \in R^{+}$and ht $\alpha<$ ht $\beta$.

Let $\tilde{\mathfrak{g}}$ be the Lie algebra of vector fields on $\Omega_{M}$ generated by the vector fields $\underline{e}_{i}$ and $\underline{f}_{i}, i=1, \ldots, n$. Recall that using the map $Y Z X$, the Lie algebra $\mathfrak{g}$ also can be viewed as a Lie algebra of vector fields on $\Omega_{M}$, generated by the vector fields $e_{i}$ and $f_{i}, i=1, \ldots, n$. Using Lemma 5.5, we obtain the following important corollary of Theorems 5.4 and 5.5.

Corollary. At every point of $\Omega_{M}$ the spaces consisting of vectors of all vector fields from $\tilde{\mathfrak{g}}$ and from $\mathfrak{g}$ coincide.

Remark. (a) As in [DCK, $\S 3.4]$ introduce the derivations $\underline{k}_{i}$ of $U_{\varepsilon}$ by

$$
\underline{k}_{i}(u)=c_{i}^{-1}\left[K_{i}^{(l)}, u\right]_{q=\varepsilon} .
$$

Then one easily checks the following formula:

$$
\underline{k}_{i}=z_{i} h_{i} / 2 \text {. }
$$

(b) The derivation $\underline{f}_{i}$ is different from the derivation $f_{i}$ introduced in [DCK, $\S 3.4]$, which (in order to avoid confusion) we denote here by $\underline{f}_{i}^{\prime}$. It follows from [DCK, (3.4.7)] that $\underline{f}_{i}=-z_{i} \underline{f}_{i}^{\prime}-y_{i} \underline{k}_{i}$, and hence $\underline{f}_{i}^{\prime}=-\frac{1}{2} y_{i} h_{i}+e_{i}$. 5.6. Recall that the quantum group $U_{M}$ and its specialization $U_{M, \varepsilon}$ are Hopf algebras with comultiplication $\Delta$, antipode $S$, and counit $\eta$ defined by

$$
\begin{gathered}
\Delta E_{i}=E_{i} \otimes 1+K_{i} \otimes E_{i}, \quad \Delta F_{i}=F_{i} \otimes K_{i}^{-1}+1 \otimes F_{i}, \quad \Delta K_{\alpha}=K_{\alpha} \otimes K_{\alpha}, \\
S E_{i}=-K_{i}^{-1} E_{i}, \quad S F_{i}=-F_{i} K_{i}, \quad S K_{\alpha}=K_{-\alpha}, \\
\eta E_{i}=0, \quad \eta F_{i}=0, \quad \eta K_{\alpha}=1 .
\end{gathered}
$$

Lemma. Let $x \in U_{M, \varepsilon}$ be such that $\Delta(x) \in Z_{\varepsilon} \otimes Z_{\varepsilon}$. Then for $s=1, \ldots, n$ one has

$$
\Delta\left(\underline{e}_{s}(x)\right)=\left(\underline{e}_{s} \otimes 1+z_{s} \otimes \underline{e}_{s}-\underline{k}_{s} \otimes z_{s} x_{s}\right) \Delta(x) .
$$

Proof. We compute in $U_{M}$,

$$
\Delta\left(\left[E_{s}^{(l)}, x\right]\right)=\left[E_{s}^{(l)} \otimes 1+K_{s}^{l} \otimes E_{s}^{(l)}+\cdots, \Delta(x)\right]
$$

where dots refer to elements that are regular at $q=\varepsilon$ and hence can be ignored. Let $\Delta(x)=\sum_{i} a_{i} \otimes b_{i}$; we have

$$
\Delta\left(\underline{e}_{s}(x)\right)=\sum_{i}\left(\left[E_{s}^{(l)}, a_{i}\right] \otimes b_{i}+\left[K_{s}^{(l)}, a_{i}\right] \otimes E_{s}^{l} b_{i}+a_{i} K_{s}^{l} \otimes\left[E_{s}^{(l)}, b_{i}\right]\right)+\cdots
$$

Specializing at $q=\varepsilon$ completes the proof.

Let $Z_{\geq 0}=Z_{0}^{0} Z_{0}^{+}$and $Z_{\leq 0}=Z_{0}^{0} Z_{0}^{-}$; these are $Q_{+}$-graded subalgebras of $Z_{0}$. For $\alpha \in R^{+}$let $Z_{\alpha}$ (resp. $Z_{\alpha}^{+}$) $=Z_{0}^{0}\left[x_{\gamma} \mid \gamma \in R^{+}, \gamma \leq\right.$ (resp. <) $\left.\alpha\right]$; we let $Z_{\alpha}^{+}=0$ if $\alpha \in \Pi$. These are $Q_{+}$-graded subalgebras of $Z_{\geq 0}$, whose $\gamma$-component coincides with that of $Z_{\geq 0}$ if $\gamma \leq$ (resp. $<$ ) $\alpha$. Similarly, we define subalgebras $Z_{-\alpha}$ and $Z_{-\alpha}^{+}$of $Z_{\leq 0}$. Let $I=\operatorname{Ker} \eta$ be the augmentation ideal of $Z_{\geq 0}$. 
Proposition. (a) $\Delta\left(x_{\alpha}\right)=x_{\alpha} \otimes z_{-\alpha}+1 \otimes x_{\alpha}+P_{\alpha}$, where $P_{\alpha} \in Z_{\alpha}^{+} \otimes Z_{\alpha}^{+}$.

(b) Let $\alpha \in R_{+}, j \in\{1, \ldots, n\}$, and let $r_{\alpha, j}=\max \left\{k \mid \alpha-k \alpha_{j} \in R_{+}\right\}$. Then for a suitable choice of $x_{\alpha}$ we have $\bmod I^{2} \otimes Z_{\geq 0}+Z_{\geq 0} \otimes I^{2}$,

$$
\begin{aligned}
\Delta x_{\alpha}= & x_{\alpha} \otimes z_{-\alpha}+1 \otimes x_{\alpha}+x_{\alpha-\alpha_{j}} \otimes x_{j} \quad \text { if } \alpha=\alpha_{i}+\alpha_{j} ; \\
\Delta x_{\alpha}= & x_{\alpha} \otimes z_{-\alpha}+1 \otimes x_{\alpha}+\sum_{i} r_{\alpha, i} x_{\alpha-\alpha_{i}} \otimes x_{i} \\
& +\sum_{\substack{\beta, \gamma \in R_{+} \backslash \Pi \\
\beta+\gamma=\alpha}} c_{\beta, \gamma} x_{\beta} \otimes x_{\gamma} \quad \text { if ht } \alpha>2,
\end{aligned}
$$

where $c_{\beta, \alpha} \in \mathbb{C}$.

(c) $Z_{ \pm \alpha}$ and $Z_{ \pm \alpha}^{+}$are Hopf subalgebras of $U_{M, \varepsilon}$.

(d) $Z_{0}, Z_{\geq 0}$, and $Z_{\leq 0}$ are Hopf subalgebras of $U_{M, \varepsilon}$.

Proof. (c) and (d) follow from (a).

We prove (a) and (b) by induction on $\alpha \in Q_{+}$.

For $\alpha \in \Pi$, (a) is clear. Otherwise, by Lemma 5.4 we have for suitable choices of $x_{\alpha}$ and $x_{\beta}$, provided that $\beta=\alpha-\alpha_{i} \in R^{+}: x_{\alpha}=p^{-1} z_{i}^{-1} \underline{e}_{i}\left(x_{\beta}\right)$. Applying Lemma 5.6, we obtain

$$
\Delta\left(x_{\alpha}\right)=p^{-1}\left(z_{i}^{-1} \otimes z_{i}^{-1}\right)\left(\underline{e}_{i} \otimes 1+z_{i} \otimes \underline{e}_{i}-\underline{k}_{i} \otimes z_{i} x_{i}\right) \Delta\left(x_{\beta}\right) .
$$

(a) follows by induction. Substituting $\Delta\left(x_{\beta}\right)$ given by the inductive assumption in (b) and using that $\underline{e}_{i}\left(z_{-\beta}\right)=-\left(\beta \mid \alpha_{i}\right) z_{-\beta+\alpha_{i}} x_{i} /\left(\alpha_{i} \mid \alpha_{i}\right)$ and $\underline{k}_{i}\left(x_{\beta}\right)=$ $\left(\beta \mid \alpha_{i}\right) x_{\beta} z_{i} /\left(\alpha_{i} \mid \alpha_{i}\right)$ proves (b).

It follows from Proposition 5.6 that $\operatorname{Spec} Z_{0}$ is a connected algebraic group and that $\operatorname{Spec} Z_{\geq 0}$ and $\operatorname{Spec} Z_{\leq 0}$ are its connected normal subgroups whose intersection is the torus $\operatorname{Spec} Z_{0}^{0}$ and whose product is $\operatorname{Spec} Z_{0}$. Let $L, L^{+}, L^{-}$, and $L^{0}$ be the Lie algebras of these groups respectively. We have $L=L^{+}+L^{-}$ (sum of ideals) and $L^{0}=L^{-} \cap L^{+}$.

We shall calculate below the structure of Lie algebras $L^{+}$and $L^{-}$. We may assume that $M=Q$. Recall that $L^{+}=\left(I / I^{2}\right)^{*}$, and that, denoting by $\bar{x}$ the class in $I / I^{2}$ of $x \in I$, the bracket in $L^{+}$can be calculated as follows $\left(\varphi_{1}, \varphi_{2} \in L^{+}, x \in I\right)$ :

$$
\left[\varphi_{1}, \varphi_{2}\right](\bar{x})=\sum_{i}\left(\varphi_{1}\left(\overline{a_{i}-\eta\left(a_{i}\right)}\right) \varphi_{2}\left(\overline{b_{i}-\eta\left(b_{i}\right)}\right)-\varphi_{1}\left(\overline{b_{i}-\eta\left(b_{i}\right)}\right) \varphi_{2}\left(\overline{a_{i}-\eta\left(a_{i}\right)}\right)\right.
$$

Here $\Delta(x)=\sum_{i} a_{i} \otimes b_{i}$, where $a_{i}, b_{i} \in Z_{\geq 0}$.

Note that by Corollary $3.4, \bar{x}_{\alpha}$ up to a sign is independent of the choice of the $x_{\alpha}$. The elements $\overline{z_{i}-1}(i=1, \ldots, n), \bar{x}_{\alpha}\left(\alpha \in R^{+}\right)$form a basis of $I / I^{2}$; let $h_{i}(i=1, \ldots, n), S_{\alpha}\left(\alpha \in R^{+}\right)$be the dual basis of $L^{+}$. In order to calculate brackets of these elements, we use Proposition 5.6(b).

A straightforward calculation shows that

$$
\left[h_{i}, S_{\beta}\right]=\frac{2\left(\omega_{i} \mid \beta\right)}{\left(\alpha_{i} \mid \alpha_{i}\right)} S_{\beta} .
$$


It follows that

$$
\begin{array}{ll}
{\left[S_{\alpha}, S_{\beta}\right]=0} & \text { if } \alpha+\beta \notin R^{+}, \\
{\left[S_{\alpha}, S_{\beta}\right]=m_{\alpha \beta} S_{\alpha+\beta}} & \text { if } \alpha+\beta \in R^{+} .
\end{array}
$$

Again, it is straightforward to show that

$$
m_{\alpha_{i}, \beta}=r_{\beta, i}+1 \text { if } \alpha_{i}+\beta \in R^{+} .
$$

Similar formulas hold for $L^{-}$. It follows that $L^{0}$ and the $S_{\alpha_{i}}$ (resp. $S_{-\alpha_{i}}$ ) generate $L^{+}$(resp. $L^{-}$) and that the Chevalley-Serre relations hold for them. Hence we obtain the following

Theorem. $L^{+}$(resp. $L^{-}$) is isomorphic to a Borel subalgebra $\mathfrak{b}$ of the Lie algebra $\mathfrak{g}$.

This result has been announced recently by N. Reshetikhin (MIT seminar). In $\S 7$ we calculate the group $\operatorname{Spec} Z_{0}$ more explicitly, along with its Poisson structure.

Remark. Let $\pi_{i} \in \operatorname{Spec} U_{M, \varepsilon}(i=1,2)$ be two irreducible representations, and let $g_{i}(i=1,2)$ be the corresponding elements of $\operatorname{Spec} Z_{0}$. It is clear that if $\pi_{1} \otimes \pi_{2}$ and $\pi_{2} \otimes \pi_{1}$ are equivalent, then $g_{1} g_{2}=g_{2} g_{1}$ in the algebraic group $\operatorname{Spec} Z_{0}$. To what extent is this condition sufficient?

\section{THE GEOMETRY OF THE QUANTUM COADJOINT ACTION}

6.1. It is proved in [DCK, $\S 3.5]$ that the derivations $\underline{e}_{i}$ (and hence $\underline{f}_{i}$ ) of the algebra $Z_{0}$ integrate to global 1-parameter groups of analytic automorphisms $\exp s \underline{e}_{i}$ (resp. $\exp s \underline{f}_{i}$ ) of the algebraic variety $\Omega_{M}=\operatorname{Spec} Z_{0}$. Denote by $\widetilde{G}$ the (infinite-dimensional) group generated by the groups $\exp s \underline{e}_{i}$ and $\exp s \underline{f}_{i}$, $i=1, \ldots, n$. The action of $\widetilde{G}$ on $\Omega_{M}$ is called the quantum coadjoint action [DCK].

Let, as before, $G^{0}=U_{-} T U_{+}$be the big cell of $G$, and let $\pi$ denote the map $Y Z X: \Omega_{M} \rightarrow G^{0}$. Recall that $\Omega_{M}=\operatorname{Spec} Z_{0}^{-} \times T \times \operatorname{Spec} Z_{0}^{+}$is an unramified (algebraic) Galois covering with the group $\frac{1}{2} M / M$. Let $F=\pi^{-1}(\operatorname{Center} G)=$ $\left\{\chi \in T \subset \Omega_{M} \mid \chi\left(z_{\beta}\right)= \pm 1\right.$ for all $\left.\beta \in Q\right\}$.

Given a conjugacy class $\mathscr{O}$ in $G$, it always intersects $G^{0}$ in a smooth connected variety $\mathscr{O}^{0}$. Theorems 5.4 and 5.5 and Corollary 5.5 immediately give us

Proposition. (a) The connected components of the variety $\pi^{-1}\left(\mathscr{O}^{0}\right)$ are orbits of the group $\widetilde{G}$.

(b) The set $F$ is the fixed point set of $\widetilde{G}$ in $\Omega_{M}$. The action of $\widetilde{G}$ in the tangent space to each point $p \in F$ induces the coadjoint action of $G$ on $\mathfrak{g}$ (after dividing by the kernel).

Proof. (b) is clear. To see (a) we use the following simple geometric fact: Let $\varphi_{i}$ $(i=1, \ldots, m)$ be global 1-parameter groups of diffeomorphisms of a connected 
manifold $M$ and let $X_{i}$ be the corresponding vector fields. Assume that the $X_{i}$ span the tangent space to $M$ at every point of $M$. Then $M$ is an orbit of the group $K$ generated by the $\varphi_{i}$. (Indeed, our assumption implies that every $K$-orbit in $M$ is open. Since $M$ is connected, the claim follows.)

Proposition 6.1(a) has a number of important consequences, some of which are solutions to some of the conjectures of [DCK]. In order to state these results, introduce some notation and terminology.

Denote by $I$ (resp. $I^{+}$) the ideal in $Z_{0}$ generated by all elements $x_{k}^{J}$ and $y_{k}^{J}$ (resp. $x_{k}^{J}$ ). Then $T=\left\{\chi \in \Omega_{M} \mid \chi(I)=0\right\}$, and we let $T_{\text {reg }}=\left\{\chi \in T \mid \chi\left(z_{\beta}\right)^{2} \neq\right.$ 1 for all $\beta \in R\}$. A $\widetilde{G}$ orbit in $\Omega_{M}$ is called unipotent if its Zariski closure contains a point from $F$. A $\widetilde{G}$-orbit in $\Omega_{M}$ is called semisimple (resp. regular semisimple) if it intersects $T$ (resp. $T_{\text {reg }}$ ).

Theorem. (a) Every $\widetilde{G}$-orbit in $\Omega_{M}$ contains an element $\chi$ such that $\chi\left(I^{+}\right)=0$.

(b) Every $\widetilde{G}$-orbit $\mathscr{O}$ in $\Omega_{M}$ is Zariski open in its Zariski closure $\mathscr{\mathscr { O }}$.

(c) $A \widetilde{G}$-orbit is closed if and only if it is semisimple.

(d) The union of all regular semisimple orbits $\widetilde{G} \cdot T_{\text {reg }}$ is Zariski open and dense in $\Omega_{M}$.

(e) There is only a finite number of unipotent orbits.

Proof. Follows from Proposition 6.1 and the well-known results on conjugacy classes in simple Lie groups [K, S].

Corollary. Every finite-dimensional irreducible representation $\rho$ of $U_{M, \varepsilon}$ in a vector space $V$ is triangularizable; i.e., there exists $\sigma \in \widetilde{G}$ and a nonzero vector $v \in V$ such that

$$
\rho\left(\sigma\left(\bar{U}_{\varepsilon}^{+}\right)\right) v=0 \quad \text { and } \rho\left(\sigma\left(K_{\beta}\right)\right) v=\lambda_{\beta} v, \quad \lambda_{\beta} \in \mathbb{C}, \beta \in M .
$$

Proof. By Theorem 6.1(a) there exists $\sigma \in \widetilde{G}$ such that $\rho\left(\sigma\left(I^{+}\right)\right)=0$. It follows that $\rho\left(\sigma\left(E_{\alpha}\right)\right)^{l}=0$ for all $\alpha \in R^{+}$. By the PBW theorem [L], it follows that $\rho\left(\sigma\left(\bar{U}_{\varepsilon}^{+}\right)\right)$consists of nilpotent endomorphisms; hence the subspace $V_{0}=\{v \in$ $\left.V \mid \rho\left(\sigma\left(\bar{U}_{\varepsilon}^{+}\right)\right) v=0\right\}$ is nonzero. Since the $K_{\beta}$ normalize $U_{\varepsilon}^{+}, V_{0}$ is $K_{\beta}^{-}$ invariant, and hence the $K_{\beta}$ have in $V_{0}$ a common eigenvector.

One can deduce from [BC, Theorem 7.1] a more precise statement than Theorem 6.1(a): Every $\widetilde{G}$-orbit in $\Omega_{M}$ contains an element $\chi$ such that

(i) $\chi\left(I^{+}\right)=0$,

(ii) the set $R_{\chi}^{+}:=\left\{\beta_{k}^{J} \in R^{+} \mid \chi\left(y_{k}^{J}\right) \neq 0\right\}$ is linearly independent (and then it is independent of $J$ ),

(iii) if $\chi\left(z_{\alpha}\right)^{2} \neq 1$ then $\alpha \notin R_{\chi}^{+}$.

6.2. We turn now to the center $Z$ of $U_{M}$ and the center $Z_{\varepsilon}$ of $U_{M, \varepsilon}$. Recall that $U_{M}=U^{0} \oplus\left(\bar{U}^{-} U_{M}+U_{M} \bar{U}^{+}\right)$and denote by $h^{\prime}$ the projection of $U_{M}$ on $U^{0}$. Similarly, define the projection $h_{\varepsilon}^{\prime}: U_{M, \varepsilon} \rightarrow U^{0}$. 
Lemma. Let $z \in Z_{\varepsilon}$ be such that

Then $z=0$.

$$
\widetilde{G} \cdot z=z \quad \text { and } \quad h_{\varepsilon}^{\prime}(z)=0 .
$$

Proof. Since the map $\operatorname{Spec} Z_{\varepsilon} \rightarrow \operatorname{Spec} Z_{0}$ (induced by inclusion) is surjective, [DCK, §3.6] due to Theorem 6.1(d), it suffices to check that the eigenvalue $\alpha$ of $z$ on each diagonal module $\bar{M}_{\varepsilon}(\lambda)$ (see [DCK, $\left.\S 3.2\right]$ ) is 0 . We have

$$
z v_{\lambda}=\alpha v_{\lambda}=u_{-} v_{\lambda}, \quad \text { where } u_{-} \in \bar{U}_{\varepsilon}^{-} .
$$

Since $u_{-}$is a nilpotent endomorphism, we see that $\alpha=0$.

Recall that the maximal torus $T$ of $G$ is the affine variety with coordinate ring $\mathbb{C}[M]$, the group ring of $M$. We identify $\mathbb{C}[M]$ with $U_{M}^{0}$ via $\beta \mapsto K_{\beta}$, $\beta \in M$. It is convenient to look at $T$ as the algebraic group $\mathbb{C}^{\times} \otimes_{\mathbb{Z}} M^{*}$. The action by left translations of $T$ on functions on $T$ can then be written as

$$
(\lambda \otimes m) K_{\beta}=\lambda^{\langle m, \beta\rangle} K_{\beta}, \quad \lambda \in \mathbb{C}^{\times}, m \in M^{*} .
$$

Consider the map of $\frac{1}{2} Q^{\vee}$ in $T$ defined by $\frac{1}{2} \gamma \mapsto(-1) \otimes \gamma$ and let $Q_{2}^{\vee}$ denote its image. One should note that $Q^{\vee} \subset M^{*}$ since $Q^{\vee}=P^{*}$ and that, for the same reason, $Q_{2}^{\vee}$ is the set of all elements of $T$ of period 2 if $G$ is simply connected (otherwise $Q_{2}^{\vee}$ may be smaller). Thus we have an action of the group $\widetilde{W}:=W \ltimes Q_{2}^{\vee}$ on $U_{M}^{0}$.

Now we can recall the construction of $Z$. Denote by $\gamma$ the automorphism of $U^{0 Q_{2}^{\vee}}$ defined by $\gamma\left(K_{\beta}\right)=q^{(\rho \mid \beta)} K_{\beta}$, and let $h=\gamma^{-1} \circ h^{\prime}$. At this point we use that $2(\rho \mid P) \subset \mathbb{Z}$. Then we have an isomorphism of algebras [DCK, Proposition 2.2(b)]

\[ h: Z \stackrel{\sim}{\rightarrow} U^{0 \tilde{W}} . \]
More explicitly, for each $\varphi \in U^{0 \widetilde{W}}$ there exists a unique
[DCK] for notation) of the form
\[ z_{\varphi}=\gamma(\varphi)+\sum_{\eta>0} \sum_{k, r \in \operatorname{Par}(\eta)} F^{k} \varphi_{k, r} E^{r}, \]

where the $\varphi_{k, r} \in U^{0}$ can be computed from a recurrent formula and $Z=$ $\left\{z_{\varphi} \mid \varphi \in U^{0 \tilde{W}}\right\}$. The recurrent formula shows that the only poles of the $\varphi_{k, r}$ are $q=$ roots of 1 and the poles of $\varphi$.

Proposition. All the $\varphi_{k, r}$ do not have a pole at $q=\varepsilon$ if $\varphi$ does not.

Proof. Let $P_{l}(q)$ be the $l$ th cyclotomic polynomial and let $m$ be the maximum of orders of poles of the $\varphi_{k, r}$. Let $z^{\prime}=P_{l}(q)^{m} z$. Then the element $\left.z^{\prime}\right|_{q=\varepsilon}$ satisfies all conditions of the lemma and is nonzero, a contradiction.

Thus, we have a well-defined injective homomorphism $\bar{h}_{\varepsilon}: U_{\varepsilon}^{0 \widetilde{W}} \rightarrow Z_{\varepsilon}$ given by $\varphi \mapsto z_{\varphi}$. Denote by $Z_{1}$ its image. (Note that it is different from that introduced in [DCK, $\S 5]$. As we shall see, all conjectures stated there are true for the $Z_{1}$, introduced here.) 
Corollary. (a) The subalgebra $Z_{1}$ of $Z_{M, \varepsilon}$ is pointwise fixed under the action of $\widetilde{G}$ and the action of $\mathscr{B}$.

(b) The center $Z_{M}$ of $U_{M}$ is pointwise fixed under the action of $\mathscr{B}$.

Proof. The first part of (a) is clear and the second part follows from (b). Now let $z \in Z_{M}$ and let $z^{\prime}:=T_{i}(z)-z \in Z_{M}$. If $z^{\prime} \neq 0$, then by Lemma 6.2 one can arrange for $z^{\prime}$ to be defined and yet $h_{\varepsilon}^{\prime}\left(z^{\prime}\right) \neq 0$. This contradicts [DCK, (5.4.2)].

6.3. We return to the study of the quantum coadjoint action. Recall that $G=$ $G_{c} / C_{G}$, where $G_{c}$ is the simply connected cover of $G$ and $C_{G}=M^{*} / Q^{\vee}$ is its fundamental group. Note that, given $m \in \mathbb{N}$, we have

$$
T_{(m)}:=\left\{\sigma \in T \mid \sigma^{m}=1\right\}=m^{-1} M^{*} / M^{*},
$$

and that $Q_{2}^{\vee} \subset T_{(2)}$ since $Q^{\vee} \subset M^{*}$. Denote by $S$ the subgroup of $T_{(2 l)}$ generated by $T_{(l)}$ and $Q_{2}^{\vee}$. We have canonical isomorphisms

$$
T_{(2 l)} / S \simeq \frac{1}{2 l} M^{*} /\left(\frac{1}{2} Q^{\vee}+\frac{1}{l} M^{*}\right) \simeq M^{*} /\left(Q^{\vee}+2 M^{*}\right) \simeq T_{(2)} / Q_{2}^{\vee} .
$$

Thus

$$
T_{(2 l)} / S \simeq C_{G} / C_{G}^{2} .
$$

Let $G^{\prime}=G_{c} / C_{G}^{2}$, so that Center $G^{\prime}=C_{G} / C_{G}^{2}$. The coroot lattice of the group $G^{\prime}$ is $Q^{\vee}+2 M^{*}$ and the weight lattice is $P \cap \frac{1}{2} M$. We list in Table 1, in the case of the adjoint group, $G=G_{\text {ad }}$ (i.e., when $C_{G}$ is the largest) the group $G^{\prime}$, its center, the groups $C_{G}$, and $C_{G} / C_{G}^{2}$.

TABLE 1.

\begin{tabular}{c|llll}
$\left(a_{i j}\right)$ & $G^{\prime}$ & Center $G^{\prime}$ & $C_{G}$ & $C_{G}^{2}$ \\
\hline$A_{2 k}$ & $G_{\mathrm{ad}}$ & 1 & $\mathbb{Z}_{2 k+1}$ & $\mathbb{Z}_{2 k+1}$ \\
$A_{2 k-1}$ & $\mathrm{SL}_{2 k} / \mathbb{Z}_{k}$ & $\mathbb{Z}_{2}$ & $\mathbb{Z}_{2 k}$ & $\mathbb{Z}_{k}$ \\
$B_{n}, C_{n}$ & $G_{c}$ & $\mathbb{Z}_{2}$ & $\mathbb{Z}_{2}$ & 1 \\
$D_{2 k}$ & $G_{c}$ & $\mathbb{Z}_{2}+\mathbb{Z}_{2}$ & $\mathbb{Z}_{2}+\mathbb{Z}_{2}$ & 1 \\
$D_{2 k-1}$ & $\mathrm{SO}_{4 k-2}$ & $\mathbb{Z}_{2}$ & $\mathbb{Z}_{4}$ & $\mathbb{Z}_{2}$ \\
$E_{6}$ & $G_{\text {ad }}$ & 1 & $\mathbb{Z}_{3}$ & $\mathbb{Z}_{3}$ \\
$E_{7}$ & $G_{c}$ & $\mathbb{Z}_{2}$ & $\mathbb{Z}_{2}$ & 1 \\
$E_{8}, F_{4}, G_{2}$ & $G_{c}$ & 1 & 1 & 1
\end{tabular}

Note that $Z_{0}^{0}=U_{\varepsilon}^{0 T_{(l)}}$; hence $U_{\varepsilon}^{0 S}$ is a subalgebra of $Z_{0}^{0}$. Consider the following subalgebra of $Z_{0}$ (which is independent of the choice of the reduced 
expression $J$ of $w_{0}$ ),

$$
Z_{0}^{\prime}=U_{\varepsilon}^{0 S}\left[x_{k}^{J}, y_{k}^{J}\right]
$$

and let $\Omega_{M}^{\prime}=\operatorname{Spec} Z_{0}^{\prime}$. The embedding $Z_{0}^{\prime} \subset Z_{0}$ induces an unramified cover $\Omega_{M} \rightarrow \Omega_{M}^{\prime}$ with the Galois group $Q_{2}^{\vee}$. Recall that the map $\Omega_{M} \rightarrow G^{0}$ is an unramified cover with the Galois group $T_{(2)}$. Also the homomorphism $G^{\prime} \rightarrow G$ is an unramified cover with the Galois group $C_{G} / C_{G}^{2} \simeq T_{(2)} / Q_{2}^{\vee}$. It follows that there exists a unique isomorphism of algebraic varieties $\tau: \Omega_{M}^{\prime} \stackrel{\sim}{\rightarrow} G^{\prime 0}$, the big cell of $G^{\prime}$, which makes the diagram

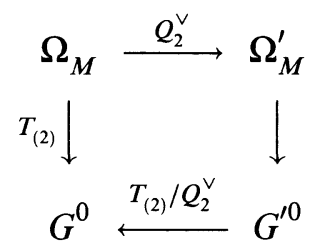

commutative. The map $\tau$ induces an embedding $\mathbb{C}\left[G^{\prime}\right] \subset Z_{0}^{\prime}$.

Proposition. (a) $Z_{0} \cap Z_{1}=\mathbb{C}\left[G^{\prime}\right]^{G^{\prime}}$, where $G^{\prime}$ acts on itself by conjugation.

(b) $Z_{0} \cap Z_{1} \subset Z_{0}^{\prime}$.

(c) $\bar{h}_{\varepsilon}\left(U_{\varepsilon}^{0 \widetilde{W}_{\varepsilon} \ltimes T_{(l)}}\right)=Z_{0} \cap Z_{1}$.

(d) The map $\operatorname{Spec} Z_{0}^{\prime} \rightarrow \operatorname{Spec} Z_{0} \cap Z_{1}$ induced by inclusion is the restriction to the big cell of the quotient morphism $G^{\prime} \rightarrow \operatorname{Spec} \mathbb{C}\left[G^{\prime}\right]^{G^{\prime}}$.

Proof. In order to prove (a) note that by the construction of $Z_{1}, h_{\varepsilon}\left(Z_{0} \cap Z_{1}\right) \subset$ $Z_{0}^{\prime}$, and also $h_{\varepsilon}\left(Z_{1}\right)=U_{\varepsilon}^{0 \widetilde{W}}$. So, if $a \in Z_{0} \cap Z_{1}$ then $h_{\varepsilon}(a) \in Z_{0}^{\prime} \cap U_{\varepsilon}^{0 \widetilde{W}}$, hence there exists a unique $\bar{a} \in \mathbb{C}\left[G^{\prime}\right]^{G^{\prime}} \subset Z_{0}^{\prime}$ such that $h_{\varepsilon}(\bar{a})=h_{\varepsilon}(a)$. (Here we use the well-known restriction isomorphism $\mathbb{C}\left[G^{\prime}\right]^{G^{\prime}} \stackrel{\sim}{\rightarrow} \mathbb{C}\left[T^{\prime}\right]^{W}$, see [S].) Since, by Proposition $6.1(\mathrm{a})$, both $a$ and $\bar{a}$ are $\widetilde{G}$-invariant, applying Lemma 6.2 , we get $a=\bar{a}$. So, $Z_{0} \cap Z_{1} \subset \mathbb{C}\left[G^{\prime}\right]^{G^{\prime}}$. Conversely, if $a \in \mathbb{C}\left[G^{\prime}\right]^{G^{\prime}}$ then $h_{\varepsilon}(a) \in$ $\left(Z_{0}^{0} \cap Z_{0}^{\prime}\right)^{W}=Z_{0}^{0 \widetilde{W}}$. But again, there exists $\bar{a} \in Z_{1}$ such that $h_{\varepsilon}(\bar{a})=h_{\varepsilon}(a)$, and by Lemma 6.2 we get $a=\bar{a} \in Z_{1}$, hence $\mathbb{C}\left[G^{\prime}\right]^{G^{\prime}} \subset Z_{0}^{\prime} \cap Z_{1}$. (b) is clear since $Z_{0} \cap Z_{1}=\mathbb{C}\left[G^{\prime}\right]^{G^{\prime}} \subset Z_{0}^{\prime}$. (c) and (d) follow immediately from (b) and the definition of $Z_{0}^{\prime}$.

Corollary. The map $\operatorname{Spec} Z_{0}^{\prime} \rightarrow \operatorname{Spec} Z_{0} \cap Z_{1}$ induced by the inclusion is smooth in codimension 1 .

Proof. Due to Proposition 6.3(d), it suffices to show that the map $\varphi^{\prime}: G^{\prime} \rightarrow$ Spec $\mathbb{C}\left[G^{\prime}\right]^{G}$ is smooth in codimension 1 . For this recall that the map $\varphi: G_{c} \rightarrow$ Spec $\mathbb{C}\left[G_{c}\right]^{G_{c}} \simeq \mathbb{C}^{n}$, given by $g \mapsto\left(\chi_{1}(g), \ldots, \chi_{n}(g)\right)$, where the $\chi_{i}$ are the characters of fundamental representations of $G_{c}$, is smooth in codimension $1[\mathrm{~S}, \S \S 5,8]$. Since $\varphi^{\prime}$ is obtained from $\varphi$ by dividing by $C_{G}^{2}$ it suffices to 
show that the quotient map of the action of $C_{G}^{2}$ on $\operatorname{Spec} \mathbb{C}\left[G_{c}\right]^{G_{c}}$ is smooth in codimension 1 . Note that $C_{G}^{2}$ is a cyclic group (see Table 1). Let $s$ denote its order and let $g$ be its generator. Let $\eta$ be a primitive $s$ th root of 1 ; then $g \chi_{j}=\eta^{s_{j}} \chi_{j}$. If $s=1$, there is nothing to prove. Otherwise, by a case-wise inspection we see that there are at least two $s_{j}$ which are relatively prime to $s$. This completes the proof.

6.4. Our next objective is to prove that $Z_{P, \varepsilon}$ is generated by its subalgebras $Z_{0}$ and $Z_{1}$. (Remark 6.4 (a) below shows that this may be false if $M \neq P$.) For this we shall prove the

Proposition. The ring $A:=\mathbb{C}[P]^{W}$ is a complete intersection over its subring $A_{l}:=\mathbb{C}[l P]^{W}$.

First, we prove a lemma. Let $P_{+}=\left\{\lambda \in P \mid\left\langle\lambda, \alpha_{i}^{\vee}\right\rangle \in \mathbb{Z}_{+}, i=1, \ldots, n\right\}$, $P_{+}^{l}=\left\{\lambda \in P_{+} \mid\left\langle\lambda, \alpha_{i}^{\vee}\right\rangle\langle l, i=1, \ldots, n\}\right.$. For $\lambda \in P_{+}$let $\chi_{\lambda}=\sum_{\mu \in W(\lambda)} e^{\mu}$. Then $\left\{\chi_{\lambda}\right\}_{\lambda \in P_{+}}$(resp. $\left.\left\{\chi_{l \lambda}\right\}_{\lambda \in P_{+}}\right)$form a $\mathbb{C}$-basis of $A$ (resp. $A_{l}$ ) and $\left\{\chi_{\lambda}\right\}_{\lambda \in P_{+}^{l}}$ form a basis of the $A_{l}$-module $A$. For $\lambda=\sum n_{i} \omega_{i} \in P_{+}$let $M_{\lambda}=\prod_{i} \chi_{\omega_{i}}^{n_{i}}$. Finally, define a partial ordering on $P_{+}$by letting $\lambda \geq \mu$ if $\lambda-\mu=\sum_{i} a_{i} \alpha_{i}$ with $a_{i} \in \mathbb{Q}, a_{i} \geq 0$.

Lemma. Let $\lambda \in P_{+}$. (a)We can write $\chi_{\lambda}=\sum_{\mu \in P_{+}^{l}} a_{\lambda \mu} \chi_{\mu}$, where $a_{\lambda \mu} \in A_{l}$ and $a_{\lambda \mu} \neq 0$ only if $\lambda \geq \mu$.

(b) We can write $M_{\lambda}=\sum_{\mu \in P_{+}^{l}} b_{\lambda \mu} \chi_{\mu}$, where $b_{\lambda \mu} \in A_{l}$ and $b_{\lambda \mu} \neq 0$ only if $\lambda \geq \mu$. Also, $b_{\lambda \lambda}=1$ if $\lambda \in P_{+}^{l}$.

(c) We can write $M_{\lambda}=\sum_{\mu \in P_{+}^{l}} c_{\lambda \mu} M_{\mu}$, where $c_{\lambda \mu} \in A_{l}$ and $c_{\lambda \mu} \neq 0$ only if $\lambda \geq \mu$.

Proof. Write $\lambda=l \lambda^{\prime}+\lambda^{\prime \prime}$, where $\lambda^{\prime} \in P_{+}, \lambda^{\prime \prime} \in P_{+}^{l}$. Then $\lambda \geq \lambda^{\prime \prime}$ and

$$
\chi_{\lambda}=\chi_{l \lambda^{\prime}} \chi_{\lambda^{\prime \prime}}+\sum_{\mu<\lambda} a_{\lambda_{\mu}}^{\prime} \chi_{\mu}, \quad \text { where } a_{\lambda \mu}^{\prime} \in \mathbb{Z} \text {. }
$$

Thus (a) follows by induction on the ordering.

Since

$$
M_{\lambda}=\chi_{\lambda}+\sum_{\mu<\lambda} b_{\lambda_{\mu}}^{\prime} \chi_{\mu}, \quad b_{\lambda \mu}^{\prime} \in \mathbb{Z},
$$

the first part of (b) follows by induction on the ordering and (a). The second part of (b) is clear.

Thus, the matrix $B:=\left(b_{\lambda \mu}\right)$ in (b) (where $\lambda, \mu \in P_{+}^{l}$ ) is invertible over $A_{l}$ and $B^{-1}=\left(c_{\lambda \mu}^{\prime}\right)$ has the same properties, i.e., we have for $\lambda \in P_{+}^{l}$

$$
\chi_{\lambda}=\sum_{\mu \leq \lambda} c_{\lambda \mu}^{\prime \prime} M_{\mu}, \quad c_{\lambda \mu}^{\prime \prime} \in A_{l}, c_{\lambda \lambda}^{\prime \prime}=1 .
$$

Substituting this in (b) we get (c). 
Proof of Proposition 6.4. By Lemma 6.4(c) we have

$$
M_{\omega_{i}}^{l}=\sum_{\mu \in P_{+}^{\prime}, \mu<l \omega_{i}} d_{i \mu} M_{\mu}, \quad d_{i \mu} \in A_{l} .
$$

Consider the polynomial algebra $A_{l}\left[x_{1}, \ldots, x_{n}\right]$ let $x_{\lambda}=x_{1}^{k_{1}} \cdots x_{n}^{k_{n}}$ for $\lambda=$ $\sum k_{i} \omega_{i}$. Let $P_{i}=x_{i}^{l}-\sum_{\mu} d_{i \mu} x_{\mu} \in A_{l}\left[x_{1}, \ldots, x_{n}\right]$, let $I=\left(P_{1}, \ldots, P_{n}\right)$ and let $B=A_{l}\left[x_{1}, \ldots, x_{n}\right] / I$. We have a surjective homomorphism $B \rightarrow A$ defined by $x_{i} \mapsto \chi_{\omega_{i}}$. We claim that it is injective. This will prove the proposition.

In order to prove the injectivity, it suffices to show that the $x_{\lambda}, \lambda \in P_{+}^{l}$, span $B$. Take $\lambda \in P_{+}$and consider the monomial $x_{\lambda}=x_{1}^{k_{1}} \cdots x_{n}^{k_{n}}$. If $k_{i}<l$ for all $i$, we are done; if not, then $k_{j}>l$ for some $j$ and we have

$$
x_{\lambda}=x_{j}^{l} x_{\lambda-l \omega_{i}}=\sum_{\mu<l \omega_{j}, \mu \in P_{+}^{l}} d_{j \mu} x_{\lambda-l \omega_{j}+\mu} .
$$

Since $\lambda-l \omega_{j}+\mu<\lambda$, the proof is completed by induction on the ordering.

Theorem. (a) $Z_{P, \varepsilon}$ is generated by $Z_{0}$ and $Z_{1}$.

(b) $Z_{P, \varepsilon}$ is an integrally closed complete intersection ring (over $Z_{0}$ ).

Proof. Let $\tilde{Z}_{P, \varepsilon}=Z_{0} \otimes_{Z_{0} \cap Z_{1}} Z_{1}$. We have natural morphisms $Z_{0} \hookrightarrow \tilde{Z}_{P, \varepsilon} \rightarrow$ $Z_{P, \varepsilon}$. In order to prove (a), it suffices to show that $\widetilde{Z}_{P, \varepsilon}$ is normal. Indeed, since $Z_{P, \varepsilon}$ is finite over $Z_{0}$ [DCK], the normality of $\tilde{Z}_{P, \varepsilon}$ implies that the map $\widetilde{Z}_{P, \varepsilon} \rightarrow Z_{P, \varepsilon}$ is injective. From [DCK, $\S 5.3$ ] it follows that this is a birational inclusion, hence an isomorphism since $Z_{P, \varepsilon}$ is normal [DCK].

Recall that

$$
Z_{1} \simeq \mathbb{C}[P]^{\widetilde{W}}=\mathbb{C}[2 P]^{W}, \quad Z_{0} \cap Z_{1}=\mathbb{C}[l P]^{\widetilde{W}}=\mathbb{C}[2 l P]^{\widetilde{W}}
$$

Hence by Proposition 6.4, $\widetilde{Z}_{P, \varepsilon}$ is a complete intersection ring over $Z_{0}$.

According to Serre's theorem [Se, Chapter 4], a complete intersection variety, which is smooth outside of a subvariety of codimension 2, is normal. Thus, in order to establish normality of $\widetilde{Z}_{P, \varepsilon}$, it suffices to show that $\operatorname{Spec} \widetilde{Z}_{P, \varepsilon}$ is smooth outside of a subvariety of codimension 2 .

It follows from (1) that Spec $\widetilde{Z}_{P, \varepsilon}$ is an open subset in an unramified covering of the fiber product

$$
X_{G}:=T / W \times_{T / W} G,
$$

where the first map $\rho_{l}: T / W \rightarrow T / W$ is induced by the $l$ th power map $\left(t \mapsto t^{l}\right)$ and the second map $\sigma: G \rightarrow T / W$ is the quotient map.

By [S, $\S \S 5,8], G=G_{1} \cup G_{2}$, where $G_{1}$ is open, $G_{2}$ is a closed subvariety of codimension $\geq 2$, and $\sigma: G_{1} \rightarrow T / W$ is a smooth map (here we use that $G$ is simply connected). Hence $T / W \times_{T / W} G_{1} \rightarrow T / W$ is a smooth map, and since $T / W$ is smooth, we obtain that $T / W \times_{T / W} G_{1}$ is smooth. Since the map 
$T / W \rightarrow T / W$ is finite, we obtain that $T / W \times_{T / W} G_{2}$ has codimension $\geq 2$ in $T / W \times_{T / W} G$, completing the proof.

(b) is clear from the previous discussion.

Remarks. (a) If $M \neq P$, the conclusion of Theorem 6.4(a) is false. For example, if $U_{Q, \varepsilon}$ is the (adjoint) quantum group of type $A_{2}$ and $\varepsilon$ is a $3 d$ root of 1 , then the element $K_{1}^{2} K_{2}$ is central but does not lie in $\widetilde{Z}_{Q, \varepsilon}$. Note also that the proof of a result similar to Theorem $6.4(\mathrm{a})$ in $[\mathrm{KW}]$ on the center of the universal enveloping algebra $U(\mathfrak{g})$ of the Lie algebra $\mathfrak{g}$ of a simple algebraic group $G$ in characteristic $p$ contains a gap. Our argument proves this result as well provided that the quotient map $\mathfrak{g}^{*} \rightarrow \mathfrak{g}^{*} / G$ is smooth in codimension 1 .

(b) We have shown that $\operatorname{Spec} Z_{P, \varepsilon}$ is an unramified cover with the Galois group $\frac{1}{2} Q^{\vee} / Q^{\vee}$ of the fiber product $G / / G \times{ }_{G / / G} G^{0}$, where the first map is the $l$ th power map and the second is the restriction of the quotient map.

(c) The proof of normality of $\operatorname{Spec} Z_{\varepsilon}$ given in [DCK] may be simplified. Namely, it is easy to prove the following fact (cf. [DCK, Proposition 1.8]): Let $C$ be an $S$-filtered algebra (where $S$ is an ordered set such that any decreasing sequence stabilizes) such that $\mathrm{GrC}$ has no zero divisors and is integrally closed. Then $C$ has these properties as well. (Indeed, it is standard that $C$ has no zero divisors. Furthermore, if $C \subset B \subset z^{-1} C$, where $B$ is a subring and $z$ is a central element of $C$, then $\bar{z}^{-1} \operatorname{Grz} B$ is a subring between $\operatorname{GrC}$ and $\bar{z}^{-1} \operatorname{GrC}$, hence coincides with $\operatorname{GrC}$. Take $x \in B \backslash C$ and let $y=z x$. Since $\bar{y}=\overline{z x}_{1}$ for some $x_{1} \in C$, we obtain an element $x-x_{1} \in B \backslash C$ such that $\operatorname{deg} z\left(x-x_{1}\right)<\operatorname{deg} y$.) Also the fact that any quasi-polynomial algebra is integrally closed is proved by the argument at the end of the proof of Proposition 1.8 of [DCK].

Corollary. (a) The action of $\widetilde{G}$ on

$$
\operatorname{Spec} Z_{P, \varepsilon}=\operatorname{Spec} Z_{1} \times_{\operatorname{Spec} Z_{0} \cap Z_{1}} \operatorname{Spec} Z_{0}
$$

extends from $\operatorname{Spec} Z_{0}$ by a trivial action on $\operatorname{Spec} Z_{1}$. Orbits of the action of $\widetilde{G}$ on $\operatorname{Spec} Z_{P, \varepsilon}$ are connected components of the preimages of orbits of $\widetilde{G}$ on $\Omega_{P}$ under the map $\tau$ : Spec $Z_{P, \varepsilon} \rightarrow \Omega_{P}$ induced by inclusion of coordinate rings.

(b) Let $x \in \Omega_{P}$ and let $\bar{x}=\sigma \circ \pi(x) \in T / W$. Recall the map $\rho_{l}: T / W \rightarrow$ $T / W$ induced by the lth power map. Then

$$
\left|\tau^{-1}(x)\right|=\left|\rho_{l}^{-1}(\bar{x})\right| .
$$

6.5. We shall give here another construction of the fiber product $G / / G \times{ }_{G / / G} G$ (cf. Remark 6.4(b)). Let $B$ be a finitely generated algebra without zero divisors, and let $A$ be a finitely generated subalgebra of $B$. Denote by $\bar{A}$ the integral closure of $A$ in $B$. This gives us a factorization of the map $\operatorname{Spec} B \rightarrow \operatorname{Spec} A$ (induced by the inclusion),

$$
\operatorname{Spec} B \rightarrow \operatorname{Spec} \bar{A} \rightarrow \operatorname{Spec} A,
$$

such that $\operatorname{Spec} \bar{A}$ is normal, the first map has connected (possibly empty) fibers, and the second map is finite, called the Stein factorization. 
Now let $G$ be a semisimple connected affine algebraic group. Given a positive integer $m$, denote by $\rho_{m}: G \rightarrow G$ the map defined by $g \mapsto g^{m}, g \in G$. Let $G \rightarrow X_{G}^{\prime} \rightarrow G$ be the Stein factorization of this map.

More explicitly this factorization can be constructed as follows (cf. §6.4). Denote by $G / / G$ the affine variety with coordinate ring the regular functions on $G$ invariant under conjugation, and let $\sigma: G \rightarrow G / / G$ be the quotient map. The map $\rho_{m}$ induces a map $\rho_{m}: G / / G \rightarrow G / / G$, so that we have a commutative diagram:

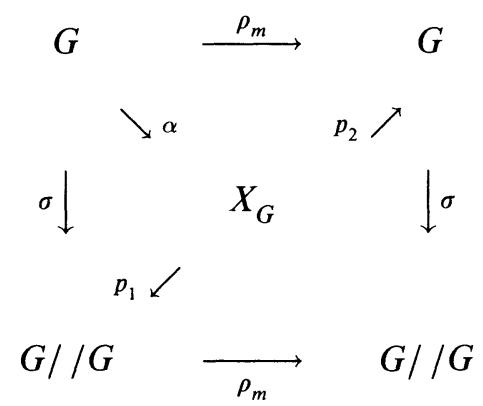

Here $X_{G}$ denotes the fiber product $G / / G \times_{G / / G} G$ and $p_{i}$ is the projection on the $i$ th factor. By the universality property of the fiber product, there exists a morphism $\alpha: G \rightarrow X_{G}$ making the diagram commutative.

Proposition. $G \stackrel{\alpha}{\rightarrow} X_{G} \stackrel{p_{2}}{\rightarrow} G$ is the Stein factorization of $\rho_{m}: G \rightarrow G$, so that $X_{G}^{\prime} \simeq X_{G}$.

Proof. Let $T$ be a maximal torus; then we have the canonical isomorphism $G / / G \stackrel{\sim}{\rightarrow} T / W$, where $W$ is the Weyl group. Since the map $\rho_{m}: T \rightarrow T$ is finite, it follows that the map $\rho_{m}: G / / G \rightarrow G / / G$ is finite, hence the map $p_{2}: X_{G} \rightarrow G$ is finite. Furthermore, it is clear that the map $\alpha: G \rightarrow X_{G}$ is birational. It remains to show that $X_{G}$ is normal. As shown in $\S 6.4$, this is the case if $G$ is simply connected. Hence it is true for arbitrary $G$.

Remark. Note that if $G$ is an arbitrary connected simply connected affine algebraic group, we denote by $\bar{G}$ its quotient by the unipotent radical, and then $X_{G}$ is the fiber product of $G$ and $X_{\bar{G}}$ over $\bar{G}$.

6.6. The orbits of the action of $\widetilde{G}$ on $\Omega_{M}$ are described by the following

Theorem. Consider the unramified cover $\pi^{\prime}: \Omega_{M} \rightarrow G^{\prime 0}$, with the Galois group $Q_{2}^{\vee}=Q^{\vee} /\left(Q^{\vee} \cap 2 M^{*}\right)$. Let $\mathscr{O}$ be a conjugacy class of a noncentral element of $G^{\prime}$ and let $\mathscr{O}_{0}=\mathscr{O} \cap G^{0}$. Then $\left(\pi^{\prime}\right)^{-1}\left(\mathscr{O}_{0}\right)$ is an orbit of $\widetilde{G}$ in $\Omega_{M}$.

Proof. We know (Proposition 6.1(a)) that connected components of $\left(\pi^{\prime}\right)^{-1}\left(\mathscr{O}_{0}\right)$ are orbits. Hence it suffices to show that $\left(\pi^{\prime}\right)^{-1}\left(\mathscr{O}_{0}\right)$ is connected. For that we have to show that the composite homomorphism $\varphi: \pi_{1}\left(\mathscr{O}_{0}\right) \rightarrow Q_{2}^{\vee}$ defined by

$$
\pi_{1}\left(\mathscr{O}_{0}\right) \stackrel{i^{*}}{\longrightarrow} \pi_{1}\left(G^{\prime 0}\right)=2 M^{*}+Q^{\vee} \rightarrow \frac{2 M^{*}+Q^{\vee}}{2 Q^{*}}=Q_{2}^{\vee}
$$

is surjective. We need the following lemma. 
Lemma. Consider the big cell in the group $\mathrm{SL}_{2}(\mathbb{C})$

$$
V=\left\{\left(\begin{array}{ll}
1 & 0 \\
y & 1
\end{array}\right)\left(\begin{array}{cc}
z & 0 \\
0 & z^{-1}
\end{array}\right)\left(\begin{array}{ll}
1 & x \\
0 & 1
\end{array}\right) \mid x, y \in \mathbb{C}, z \in \mathbb{C}^{\times}\right\},
$$

and let $a \in \mathbb{C}$. Let $V_{a}$ denote the intersection with $V$ of the conjugacy class of noncentral elements of $\mathrm{SL}_{2}(\mathbb{C})$ with trace $a$. Then the inclusion map induces $a$ surjective homomorphism of fundamental groups $\pi_{1}\left(V_{a}\right) \rightarrow \pi_{1}(V)$.

Proof. The set $V_{a}$ is a hypersurface in $V$ given by the equation $z+z^{-1}+z x y=$ $a$, with the point $(0,0, \pm 1)$ deleted if $a= \pm 2$. Consider the $m$ th cover $\sigma_{m}: \operatorname{Spec} \mathbb{C}\left[x, y, t, t^{-1}\right] \rightarrow V=\operatorname{Spec} \mathbb{C}\left[x, y, z, z^{-1}\right]$ given by $(x, y, t) \mapsto$ $\left(x, y, t^{m}\right)$. Note that $\sigma_{m}^{-1}\left(V_{a}\right)$ has equation $t^{m}+t^{-m}+t^{m} x y=a$, which is irreducible in $\mathbb{C}\left[x, y, t, t^{-1}\right]$. Since $\pi_{1}(V)=\mathbb{Z}$, this proves the lemma.

Now we complete the proof of Theorem 6.6. Since our claim holds for $\mathscr{O}$, if it holds for an orbit in its closure, it suffices to consider two cases (since the closure of $\mathscr{O}$ contains a semisimple conjugacy class):

(a) $\mathscr{O}$ is a conjugacy class of a noncentral semisimple element $\exp 2 \pi i h$, $h \in \mathfrak{h}$.

(b) $\mathscr{O}$ is a conjugacy class of a nontrivial unipotent element.

In case (a) there exists a long root $\alpha$ such that $\left(\alpha^{\vee} \mid h\right) \notin \mathbb{Z}$, where $\alpha^{\vee}=$ $2 \alpha /(\alpha \mid \alpha)$. Let $\gamma_{\alpha}: \mathrm{SL}_{2}(\mathbb{C}) \rightarrow G^{\prime}$ be the homomorphism corresponding to $\alpha$. It is well known that $\gamma_{\alpha}$ is injective (see, e.g., [KW, Proposition 2.1]). Then $\mathscr{O} \cap \gamma_{2}\left(\mathrm{SL}_{2}(\mathbb{C})\right)$ is a noncentral conjugacy class in $\mathrm{SL}_{2}(\mathbb{C})$. Due to the lemma, this implies that the image of $i^{*}$ contains $\alpha^{\vee}$. Using the Weyl group, we see that the image of $i^{*}$ contains $\alpha^{\vee}$ for each long root $\alpha$, hence contains $Q^{\vee}$, and hence the map $\varphi$ is surjective. In case (b), it suffices to look at the conjugacy class $\mathscr{O}$ of $\exp e_{\alpha}$, where $e_{\alpha}$ is a root vector attached to a long root $\alpha$, since it is well known that the closure of any nontrivial unipotent conjugacy class contains this one (since the projectivization of the orbit of $e_{\alpha}$ in $\mathfrak{g}$ is the only closed $G$-orbit in $\mathbb{P g}$ ). The same argument as that in case (a) now completes the proof.

Corollary. (a) Two elements of $T \backslash F$ lie in the same orbit of $\widetilde{G}$ if and only if they lie in the same orbit of $\widetilde{W}$.

(b) The closure of a $\widetilde{G}$-orbit $\mathscr{O}$ of a nonunipotent point in $\Omega_{M}$ contains a unique closed $\widetilde{G}$-orbit, which we denote by $\mathscr{O}_{s}$.

6.7. In this section we study the invariants of the action of $\widetilde{G}$ on $\Omega_{M}$.

Theorem. (a) The restriction homomorphism induced by inclusion $i: T \rightarrow \Omega_{M}$ gives an isomorphism of the algebras of invariants

$$
i^{*}: Z_{0}^{\widetilde{G}} \stackrel{\sim}{\longrightarrow} Z_{0}^{0 \widetilde{W}} .
$$

(b) The map $h_{\varepsilon}$ induces an isomorphism $Z_{M, \varepsilon}^{\widetilde{G}} \simeq U_{\varepsilon}^{0 \widetilde{W}}$.

(c) $Z_{1}=Z_{M, \varepsilon}^{\widetilde{G}}$. 
Proof. First of all, the homomorphism $i^{*}: Z_{\tilde{\sigma}}^{\widetilde{G}} \rightarrow U_{\tilde{\widetilde{W}}^{0}}^{0}$ is injective by Lemma 6.2. Furthermore, by Corollary $6.6(\mathrm{a}), i^{*}: Z_{0}^{\widetilde{G}} \rightarrow Z_{0}^{0 \widetilde{W}}$. This homomorphism induces an isomorphism of the fields of fractions. Indeed, consider the composite map $\operatorname{Spec} Z_{0} \rightarrow G^{0} \rightarrow T / W$. Using Proposition 6.1(a) and the Stein factorization we see that there exists a Zariski open $\widetilde{G}$-invariant subset $C^{\prime} \subset \operatorname{Spec} Z_{0}$ and a morphism of $C^{\prime}$ to some algebraic variety whose fibers consist of $\widetilde{G}$ orbits. It follows from Corollary 6.5(a) that the latter variety is birationally isomorphic to $T / \widetilde{W}$, proving the claim.

Finally, $i^{*}\left(Z_{0}^{\widetilde{G}}\right)=Z_{0}^{0 \widetilde{W}}$. Indeed, if $p \in Z_{0}^{0 \widetilde{W}}$, by the above, there exists $Q$ in the field of fractions of $Z_{0}^{\widetilde{G}}$ such that $i^{*}(Q)=p$. Suppose that $Q \notin Z_{0}^{\widetilde{G}}$. Since $Z_{0}$ is normal, we can write $Q=p_{1} / p_{2}$ in such a way that there exists $a \in \operatorname{Spec} Z_{0}$ such that $p_{1}(a) \neq 0$, but $p_{2}(a)=0$. Also, clearly, $g \cdot p_{i}=\varphi(g) p_{i}$, $g \in \widetilde{G}$, where $\varphi(g) \in Z_{0}^{0 \times}$. Hence the set of zeros of $p_{2}$ in $\operatorname{Spec} Z_{0}$ is $\widetilde{G}$ invariant; let $\mathscr{O}$ be a closed $\widetilde{G}$-orbit in this set (it exists by Proposition $6.1(\mathrm{a})$ by taking an orbit of minimal dimension). By Theorem $6.1(\mathrm{c}), \mathscr{O} \cap T \neq \varnothing$, hence $p$ is not regular on $T$, a contradiction. This proves (a).

(b) follows from (a) by the same arguments as in [KW, §5]. Finally since $Z_{1} \subset$ $Z_{M, \varepsilon}^{\widetilde{G}}$ (by Corollary 6.2(a)) and $h_{\varepsilon}\left(Z_{1}\right)=U_{\varepsilon}^{0 \widetilde{W}}$, by Lemma 6.2 we conclude that $Z_{1}=Z_{M, \varepsilon}^{\widetilde{G}}$, proving (c).

Remark. (a) This theorem may be used to write down explicit equations for the $z_{\varphi}$. Consider the homomorphism $f_{l}: U_{\varepsilon}^{0} \rightarrow U_{\varepsilon}^{0}$ given by $f_{l}\left(K_{\beta}\right)=K_{l \beta}$ and recall the identification of $T$ with $\operatorname{Spec} U_{\varepsilon}^{0}$. It is easy to see that for each $\varphi \in U_{\varepsilon}^{0}$ there exists a unique $\tilde{\varphi}_{1} \in U_{\varepsilon}^{0}$ such that

$$
\tilde{\varphi}:=\prod_{g \in T_{l}}(\varphi-\varphi(g))=\tilde{\varphi}_{1} \circ f_{l} .
$$

If $\varphi \in U_{\varepsilon}^{0 \widetilde{W}}$, it follows from the theorem that $z_{\tilde{\varphi}_{1}} \in Z_{0}$ and that $z_{\varphi}$ satisfies

$$
\prod_{g \in T_{l}}\left(z_{\varphi}-\gamma(\varphi)(g)\right)=z_{\tilde{\varphi}_{1}} .
$$

Note that in the case of $U_{q}\left(s l_{2}\right)$ this equation appears in [DCK, $\S 4$ ].

(b) It follows from Theorem 6.7 that $Z_{1} \cap Z_{P, 0}$ is a polynomial algebra on $n$ generators $Q_{1}, \ldots, Q_{n}$ constructed as follows. Let $\sigma_{j}$ be the $j$ th fundamental representation of the group $G_{P}$ in a vector space $V_{j}$. Then $Q_{j}(a)=$ $\operatorname{tr}_{V_{j}} \sigma_{j}(\pi(a)), a \in \operatorname{Spec} Z_{P, 0}$.

6.8. In conclusion of this section, we present a conjecture (similar to that in [WK] on representations of Lie algebras in characteristic $p$ ) and state a result (similar to Theorem 2 from [WK]) that confirms this conjecture.

Let $\sigma$ be a finite-dimensional irreducible representation of the quanturn group $U_{M, \varepsilon}$ in a vector space $V$. Let $\chi_{\sigma}: Z_{0} \rightarrow \mathbb{C}$ be the corresponding point of $\Omega_{M}$, and let $\mathscr{O}$ be the $\widetilde{G}$-orbit of $\chi_{\sigma}$. 
Conjecture. $\operatorname{dim} V$ is divisible by $l^{(\operatorname{dim} \mathscr{O}) / 2}$. In particular, $\operatorname{dim} V=l^{N}$ if $\mathscr{O}$ is an orbit of maximal dimension.

Let $g \in \widetilde{G}$ be such that $\chi:=g \chi_{\sigma}$ has the property that $\chi\left(I^{+}\right)=0$ (see Theorem 6.1(a)). Define $\chi_{s} \in \Omega_{M}$ by $\chi_{s}\left(z_{\beta}\right)=\chi\left(z_{\beta}\right), \beta \in M$, and $\chi_{s}(I)=0$, so that $\pi\left(\chi_{s}\right) \in T$ (and $\mathscr{O}_{s}=\widetilde{G} \cdot \chi_{s}$ ). Let $R^{\sigma}$ be the set of $\alpha \in R$ that vanishes on the Lie algebra of the center of the centralizer of $\pi\left(\chi_{s}\right)$. We call $\sigma$ a representation of parabolic type if $R^{\sigma} \neq R$ (i.e., the center of the centralizer of $\pi\left(\chi_{s}\right)$ in $G$ is infinite). In this case we may assume that $g \in \widetilde{G}$ is such that $R^{\sigma}:=\mathbb{Z} \Pi^{\prime} \cap R$ where $\Pi^{\prime}$ is a subset of $\Pi$ different from $\Pi$. Let $M^{\prime}=\mathbb{Z} \Pi^{\prime}$ and let $U_{M^{\prime}, \varepsilon}^{\prime}$ denote the subalgebra of $U_{M, \varepsilon}$ generated by $U_{\varepsilon}^{0}$ and the $E_{i}$ and $F_{i}$ such that $\alpha_{i} \in \Pi^{\prime}$, and let $U^{\sigma}=U_{M^{\prime}, \varepsilon}^{\prime} U_{\varepsilon}^{+}$be the corresponding "parabolic" subalgebra. The proof of the following result is essentially the same as that of Theorem 2 from [WK]:

Theorem [DCK1]. Let $\sigma$ be an irreducible representation of parabolic type of $U_{M, \varepsilon}$ in a finite-dimensional vector space $V$. Then $V$ contains a unique irreducible $U^{\sigma}$-submodule $V^{\prime}$ (which is in fact a $U_{\varepsilon}^{\prime}$-module), and the $U_{M, \varepsilon}$-module $V$ is induced from the $U^{\sigma}$-module $V^{\prime}$. In particular, $\operatorname{dim} V=l^{t / 2} \operatorname{dim} V^{\prime}$, where $t=\left|R \backslash R^{\sigma}\right|$.

Remarks. (a) The irreducible $U_{M, \varepsilon}^{\prime}$-module $V^{\prime}$ is a representation of nonparabolic type. Thus, Theorem 6.8 reduces the representation theory of $U_{M, \varepsilon}$ to the study of representations of nonparabolic type.

(b) If $\sigma$ is not of parabolic type, then $\pi\left(\chi_{s}\right)$ is conjugate in $G$ to an element of the form $\sigma_{m} u$, where $u$ is a unipotent element of $G$ commuting with $\sigma_{m}$ and $\sigma_{m}$ is a finite order element of $G$ such that $\left(\operatorname{Ad} \sigma_{m}\right) e_{j}=e_{j}$ and $\left(\operatorname{Ad} \sigma_{m}\right) f_{j}=f_{j}$ for $m \neq j,\left(\operatorname{Ad} \sigma_{m}\right) e_{m}=\left(\exp \left(2 \pi i / a_{m}\right)\right) e_{m},\left(\operatorname{Ad} \sigma_{m}\right) f_{m}=$ $\left(\exp \left(-2 \pi i / a_{m}\right)\right) f_{m}$. Here $\sum_{j=1}^{n} a_{j} \alpha_{j}$ is the decomposition of the highest root and $m=0,1, \ldots, n$.

(c) In the $A_{n}$ case (and only in that case) all nonparabolic representations are unipotent (i.e., $\chi_{s}$ is unipotent).

(d) If $\chi(\sigma) \notin F$, then $\operatorname{dim} V$ is divisible by $l^{n}$. Indeed, using $\widetilde{G}$, we can make all $x_{i}(\chi(\sigma))$ nonzero and hence all the $E_{i}$ invertible. Then all weight spaces with respect to $U^{0}$ have the same dimension.

\section{7. $\Omega_{M}$ AS A Poisson Algebraic group}

7.1. Recall that a Poisson bracket on a commutative algebra $A$ is a bilinear map

$$
\{,\}: A \wedge A \rightarrow A
$$

that satisfies the Jacobi identity and for which $\{a b, c\}=a\{b, c\}+\{a, c\} b$.

Given two commutative algebras with a Poisson bracket, $A$ and $B$, one defines a Poisson bracket on $A \otimes B$ by

$$
\left\{a_{1} \otimes b_{1}, a_{2} \otimes b_{2}\right\}=\left\{a_{1}, a_{2}\right\} \otimes b_{1} b_{2}+a_{1} a_{2} \otimes\left\{b_{1}, b_{2}\right\} .
$$


A commutative Hopf algebra $A$ with comultiplication $\Delta$, antipode $S$, and counit $\eta$ is called a Poisson Hopf algebra if $\Delta: A \rightarrow A \otimes A$ and $\eta: A \rightarrow k$ are homomorphisms of Poisson algebras.

Let $\Delta^{\prime}=p \circ \Delta$, where $p: A \otimes A \rightarrow A \otimes A$ is the permutation map, and let

$$
\delta=\Delta-\Delta^{\prime}: A \rightarrow A \wedge A .
$$

Let $\mathfrak{m}=\operatorname{Ker} \eta$. Then $\delta(\mathfrak{m}) \subset \mathfrak{m} \wedge \mathfrak{m}$ since

$$
\delta(a)=\sum_{i}\left(\left(a_{i}-\eta\left(a_{i}\right)\right) \otimes\left(b_{i}-\eta\left(b_{i}\right)\right)\right)-\left(\left(b_{i}-\eta\left(b_{i}\right)\right) \otimes\left(a_{i}-\eta\left(a_{i}\right)\right)\right),
$$

where $\Delta(a)=\sum_{i} a_{i} \otimes b_{i}$. It follows that $\delta\left(\mathfrak{m}^{2}\right) \subset \mathfrak{m}^{2} \wedge \mathfrak{m}^{2}$, hence $\delta$ induces a map $\mathfrak{m} / \mathfrak{m}^{2} \rightarrow\left(\mathfrak{m} / \mathfrak{m}^{2}\right) \wedge\left(\mathfrak{m} / \mathfrak{m}^{2}\right)$, which is again denoted by $\delta$. This is a Lie comultiplication map, i.e., the contragredient map defines a Lie algebra structure on $\left(\mathfrak{m} / \mathfrak{m}^{2}\right)^{*}$.

On the other hand, $\{\mathfrak{m}, \mathfrak{m}\} \subset \mathfrak{m}$, hence $\left\{\mathfrak{m}^{2}, \mathfrak{m}^{2}\right\} \subset \mathfrak{m}^{2}$. Thus the Poisson bracket on $A$ induces a Lie bracket on $\mathrm{m} / \mathrm{m}^{2}$. The property that $\Delta$ is a homomorphism of Poisson algebras implies that $\delta$ is a 1-cocycle for the Lie algebra $\mathfrak{m} / \mathfrak{m}^{2}$ acting in the usual way on $\left(\mathfrak{m} / \mathfrak{m}^{2}\right) \wedge\left(\mathfrak{m} / \mathfrak{m}^{2}\right)$.

Thus we obtain a Lie bialgebra $L(A)=\left(\mathfrak{m} / \mathfrak{m}^{2}\right)^{*}$ associated to the Poisson Hopf algebra $A$ (cf. [D]).

If $A$ is a finitely generated commutative Poisson Hopf algebra, then $\operatorname{Spec} A$ is an algebraic group called a Poisson algebraic group. Then $L(A)$ is called the Lie bialgebra of $\operatorname{Spec} A$ (these notions were introduced by Drinfeld [D] in the category of Lie groups).

\subsection{We shall need the following simple}

Lemma. Let $A$ and $B$ be two commutative Poisson Hopf algebras, and let $\varphi: A \rightarrow B$ be an algebra isomorphism. Let $a_{1}, \ldots, a_{s}$ be a set of Poisson generators of $A$ (i.e., $A$ equals the smallest Poisson subalgebra containing all the $\left.a_{i}\right)$. Suppose that

(i) $\varphi\left(\eta_{A}(a)\right) k=\eta_{B}(\varphi(a)), a \in A$;

(ii) $\varphi \otimes \varphi\left(\Delta_{A}\left(a_{i}\right)\right)=\Delta_{B} \varphi\left(a_{i}\right), i=1, \ldots, s$;

(iii) $\left\{\varphi\left(a_{i}\right), \varphi(a)\right\}=\varphi\left(\left\{a_{i}, a\right\}\right), i=1, \ldots, s, a \in A$.

Then $\varphi$ is an isomorphism of Poisson Hopf algebras.

Proof. Due to the Jacobi identity, (iii) implies that $\varphi$ is a Poisson algebra isomorphism. Now, due to the compatibility of $\Delta$ and $\eta$ with $\{$,$\} , conditions$ (i) and (ii) imply that $\varphi$ is a Hopf algebra isomorphism.

7.3. Recall (see $\S 5$ ) that $Z_{0, M}$ is a finitely generated commutative Hopf algebra. We give it a structure of a Poisson Hopf algebra by a usual formula (the choice of its normalization will become clear later)

$$
\{a, b\}=(\hat{a} \hat{b}-\hat{b} \hat{a}) / l\left(q^{l}-q^{-l}\right) \bmod (q-\varepsilon) .
$$

Here $\hat{a}$ stands for a preimage of $a \in Z_{0}$ in $U_{M, \mathscr{A}}$ under the canonical homomorphism $\varphi_{\varepsilon}: U_{M, \mathscr{A}} \rightarrow U_{M, \varepsilon}(\Delta$ and $\eta$ are Poisson algebra homomorphisms 
since $\Delta: U_{M, \mathscr{A}} \rightarrow U_{M, \mathscr{A}} \otimes U_{M, \mathscr{A}}$ and $\eta: U_{M, \mathscr{A}} \rightarrow \mathscr{A}$ are algebra homomorphisms).

Remark. (a) Let $N_{\varepsilon}=\varphi_{\varepsilon}^{-1}\left(Z_{\varepsilon}\right)$. Given $u \in N_{\varepsilon}$ and $v \in U_{\varepsilon}$, let

$$
P_{u}(v)=(u \hat{v}-\hat{v} u) /(q-\varepsilon) \bmod (q-\varepsilon) .
$$

The map $u \rightarrow P_{u}$ defines a representation of $\bar{N}_{\varepsilon}:=N_{\varepsilon} /(q-\varepsilon)^{2} U_{\varepsilon}$ (viewed as a Lie algebra) by derivations of $U_{\varepsilon}$. (Restricting to $Z_{\varepsilon}$ we recover, up to a constant factor, the Poisson bracket.) We have the following exact sequence of Lie algebras

$$
0 \rightarrow U_{\varepsilon} \stackrel{q-\varepsilon}{\longrightarrow} \bar{N}_{\varepsilon} \stackrel{\varphi_{\varepsilon}}{\longrightarrow} Z_{\varepsilon} \rightarrow 0 .
$$

(b) Denote by $\widetilde{\widetilde{G}}$ the group generated by all convergent series $\exp D, D \in$ $\bar{N}_{\varepsilon}$. Since the orbits of $\widetilde{\widetilde{G}}$ in $\Omega_{M}$ are connected, it follows from Proposition 6.1(a) that the orbit decompositions for $\widetilde{G}$ and $\widetilde{\widetilde{G}}$ in $\Omega_{M}$ are the same.

7.4. Here we recall (in a convenient to us form) an important example of a Poisson Hopf algebra (cf. [STS, D, LuR] and references therein). In the next sections we will show that this Poisson Hopf algebra is isomorphic to the Poisson Hopf algebra $Z_{0, M}$ considered in $\S 7.3$.

We keep the notation of $\S 4.1$. In particular, $\mathfrak{g}$ is a simple Lie algebra corresponding to the Cartan matrix $\left(a_{i j}\right), G$ is a connected algebraic group with Lie algebra $\mathfrak{g}$ and such that the group of characters of a maximal torus $T$ is $M, U_{+}$, and $U_{-}$are unipotent subgroups of $G$ corresponding to positive and negative roots, so that $G^{0}=U_{-} T U_{+}$is the big cell of $G$, etc. We normalize an invariant symmetric bilinear form $(\cdot \mid \cdot)$ on $\mathfrak{g}$ by the condition that it induces the bilinear form $(\cdot \mid \cdot)$ on $Q$ defined in $\S 0.2$ (i.e., the square length of a short root in 2). When restricted to a subalgebra $\mathbb{C} e_{i}+\mathbb{C} f_{i}+\mathbb{C} h_{i}$, this becomes the usual trace form on $\operatorname{sl}_{2}(\mathbb{C})$ multiplied by $d_{i}^{-1}$.

Consider the group $G \times G$ and the following two subgroups

$$
\widetilde{\Omega}=\left\{\left(t^{-1} u_{-}, t u_{+}\right) \mid t \in T, u_{ \pm} \in U_{ \pm}\right\}, \quad K=\{(g, g) \mid g \in G\} .
$$

Consider the (nondegenerate invariant) bilinear form on $\mathfrak{g} \oplus \mathfrak{g}=\operatorname{Lie}(G \times G)$

$$
\left(\left(x_{1}, x_{2}\right) \mid\left(y_{1}, y_{2}\right)\right)=-\left(x_{1} \mid y_{1}\right)+\left(x_{2} \mid y_{2}\right) \text {. }
$$

With respect to this form, $\mathfrak{g} \oplus \mathfrak{g}$, Lie $\widetilde{\Omega}$, and Lie $K$ form a Manin triple, i.e., Lie $\widetilde{\Omega}$ and Lie $K$ are isotropic subalgebras whose sum is $\mathfrak{g} \oplus \mathfrak{g}$. This allows us to identify $(\operatorname{Lie} \widetilde{\Omega})^{*}$ with $\operatorname{Lie} K$.

Consider the map $\tilde{\pi}: \widetilde{\Omega} \rightarrow G$ defined by $\tilde{\pi}(a, b)=a^{-1} b$. Note that $\tilde{\pi}$ is an unramified cover of the big cell $G^{0}$ of $G$.

Given an affine algebraic manifold $X$ we denote by $\mathbb{C}[X]$ (resp. Vect $X$, resp. $\mathscr{D} X)$ the space of regular functions (resp. vector fields, resp. differential 1 -forms) on $X$. To define a Poisson bracket $\{$,$\} on \mathbb{C}[X]$ is equivalent to defining a homomorphism of $\mathbb{C}[X]$-modules $\tau: \mathscr{D} X \rightarrow \operatorname{Vect} X$ (satisfying certain conditions), so that $\{f, g\}=\langle\tau(d f), d g\rangle$. 
We define the map $\tau: \mathscr{D} \widetilde{\Omega} \rightarrow \operatorname{Vect} \widetilde{\Omega}$ as follows. We identify $(\operatorname{Lie} \widetilde{\Omega})^{*}$ with the space of left-invariant 1 -forms on $\widetilde{\Omega}$. On the other hand, the adjoint action of $G$ on itself gives an embedding Lie $G \subset \operatorname{Vect} G$. This gives a linear map $\tau_{0}:(\operatorname{Lie} \widetilde{\Omega})^{*} \rightarrow \operatorname{Vect} G$. Since $\tilde{\pi}$ is unramified we have a map $\tilde{\pi}^{*}: \operatorname{Vect} G \rightarrow$ Vect $\widetilde{\Omega}$. Then $\tau$ is the homomorphism of $\mathbb{C}[\widetilde{\Omega}]$-modules defined by the linear map $\tilde{\pi}^{*} \circ \tau_{0}$.

One checks as in [LuR] that $\tau$ defines a Poisson bracket on $\mathbb{C}[\widetilde{\Omega}]$ making $\mathbb{C}[\widetilde{\Omega}]$ a Poisson Hopf algebra. (In fact our setup is a complexification of that of [LuR].)

7.5. We keep using the notation of 4.1. Let

$$
\mathfrak{n}_{ \pm}=\bigoplus_{\alpha \in R^{+}} \mathfrak{g}_{ \pm \alpha}, \quad \mathfrak{n}_{ \pm}^{(i)}=\bigoplus_{\alpha \in R^{+} \backslash\left\{\alpha_{i}\right\}} \mathfrak{g}_{ \pm \alpha},
$$

so that $\mathfrak{n}_{+}=\mathfrak{n}_{+}^{(i)}+\mathbb{C} e_{i}$ and $\mathfrak{n}_{-}=\mathfrak{n}_{-}^{(i)}+\mathbb{C} f_{i}(i=1, \ldots, n)$. We have the corresponding semidirect product of groups

$$
U_{+}=U_{+}^{(i)} \ltimes \exp \mathbb{C} e_{i}, \quad U_{-}=U_{-}^{(i)} \ltimes \exp \mathbb{C} f_{i} .
$$

This allows us to define regular functions $\tilde{x}_{i}$ and $\tilde{y}_{i}$ on $U_{+}$and $U_{-}$respectively by letting

$$
u_{+}=u_{+}^{(i)} \exp -\tilde{x}_{i} e_{i}, \quad u_{-}=u_{-}^{(i)} \exp \tilde{y}_{i} f_{i}, \quad \text { where } u_{ \pm}^{(i)} \in U_{ \pm}^{(i)} .
$$

Recall that $\mathbb{C}[T]=M$, i.e., any $\alpha \in M$ defines a regular function on $T$, which we denote by $\tilde{z}_{\alpha}$. We extend these functions to the whole $\widetilde{\Omega}$ by letting $\tilde{x}_{i}, \tilde{y}_{i}$, and $\tilde{z}_{\alpha}$ equal on $\left(t^{-1} u_{-}, t u_{+}\right)$to $\tilde{x}_{i}\left(u_{+}\right), \tilde{y}_{i}\left(u_{-}\right)$, and $\alpha(t)$ respectively.

Define the regular automorphisms $\widetilde{T}_{i}$ of the algebraic variety $\widetilde{\Omega}$,

$$
\widetilde{T}_{i}\left(t^{-1} u_{-}^{-1}, t u_{+}\right)=\left(t_{i} t^{-1}\left(u_{-}^{(i)}\right)^{-1}\left(\exp \tilde{x}_{i} e_{i}\right) t_{i}^{-1}, t_{i} t^{-1}\left(\exp \tilde{y}_{i} f_{i}\right) t^{2} u_{+}^{(i)} t_{i}^{-1}\right) \text {, }
$$

where the $t_{i}$ are defined in $\S 4.1$.

Finally, define a map $\varphi: \Omega_{M} \rightarrow \widetilde{\Omega}_{M}$ as follows. Recall that $\Omega_{M}=\operatorname{Spec} Z_{0}=$ $\operatorname{Spec} Z_{0}^{-} \times \operatorname{Spec} Z_{0}^{0} \times \operatorname{Spec} Z_{0}^{+}$and that $\operatorname{Spec} Z_{0}^{0}=T$. Then $\varphi=\varphi_{-} \times \varphi_{0} \times \varphi_{+}$, where

$$
\varphi_{-}\left(u_{-}\right)=\left(Y\left(u_{-}\right)^{-1}, 1\right), \quad \varphi_{+}\left(u_{+}\right)=\left(1, X\left(u_{+}\right)\right), \quad \varphi_{0}(t)=\left(t^{-1}, t\right) .
$$

Here $X$ and $Y$ are maps defined in $\S 4.4$. By the definitions, we have $\pi=\tilde{\pi} \circ \varphi$. 7.6. Theorem. (a) $\varphi$ is an isomorphism of Poisson algebraic groups.

(b) The $\widetilde{T}_{i}$ satisfy the braid relations and the map $\varphi^{*}$ is a $\mathscr{B}$-algebra isomorphism such that $\varphi^{*}(\tilde{y})=y_{i}, \varphi^{*}\left(\tilde{x}_{i}\right)=x_{i}, \varphi^{*}\left(\tilde{z}_{\alpha}\right)=z_{\alpha}$.

Proof. We apply Lemma 7.2 to the map $\varphi^{*}: \mathbb{C}[\widetilde{\Omega}] \rightarrow \mathbb{C}\left[\Omega_{M}\right]$ and the elements $\tilde{x}_{i}, \tilde{y}_{i}(i=1, \ldots, n), \tilde{z}_{\alpha}(\alpha \in M)$. It is clear that $\Delta \tilde{z}_{\alpha}=\tilde{z}_{\alpha} \otimes \tilde{z}_{\alpha}$. Since $\exp \mathbb{C} e_{i}$ and $T$ normalize $U_{+}^{(i)}$, it suffices to calculate $\Delta$ in the subgroup $\left\{\left(t^{-1}, t \exp -\tilde{x}_{i} e_{i}\right)\right\}$ of $\widetilde{\Omega}$, which gives

$$
\Delta \tilde{x}_{i}=1 \otimes \tilde{x}_{i}+\tilde{x}_{i} \otimes \tilde{z}_{-\alpha_{i}} .
$$


Similarly we have

$$
\Delta \tilde{y}_{i}=1 \otimes \tilde{y}_{i}+\tilde{y}_{i} \otimes \tilde{z}_{-\alpha_{i}} .
$$

Thus the assumption (ii) of Lemma 7.2 holds. Since assumption (i) holds trivially, it remains to show that assumption (iii) holds. For $\tilde{a} \in \widetilde{\Omega}$ (resp. $a \in \Omega_{M}$ ) let $\widetilde{P}_{\tilde{a}}$ (resp. $P_{a}$ ) be the operators on $\mathbb{C}[\widetilde{\Omega}]$ (resp. $\mathbb{C}\left[\Omega_{M}\right]$ ) defined by $\widetilde{P}_{\tilde{a}}(u)=\{\tilde{a}, u\}$ (resp. $\left.P_{a}(u)=\{a, u\}\right)$. We have to show that these operators correspond to each other, i.e., that $\varphi^{*} \circ \widetilde{P}_{\tilde{a}}=P_{a} \circ \varphi^{*}$, for $\tilde{a}=\tilde{x}_{i}, \tilde{y}_{i}$, and $\tilde{z}_{a}$.

Let $\tilde{\mathfrak{m}}=\operatorname{Ker} \eta \subset \mathbb{C}[\widetilde{\Omega}]$ and let $\bar{x}_{i}$ be the image of $\tilde{x}_{i}$ in $\tilde{\mathfrak{m}} / \tilde{\mathfrak{m}}^{2}=$ Lie $K$. As above, it is clear that $\bar{x}_{i}=c_{i}\left(f_{i}, f_{i}\right)$, for some $c_{i} \in \mathbb{C}$. Since

$$
\bar{x}_{i}\left(e_{i}\right)=-1 \text { and }\left(\left(f_{i}, f_{i}\right) \mid\left(0, e_{i}\right)\right)=d_{i}^{-1} \text {, }
$$

we deduce that $\bar{x}_{i}=-d_{i}\left(f_{i}, f_{i}\right)$.

Now we apply the left translation $L_{g}$ to $\bar{x}_{i}, g \in \widetilde{\Omega}$. Let $\mathfrak{m}_{g}=\{u \in$ $\mathbb{C}[\widetilde{\Omega}] \mid u(g)=0\}$. Recall that if $u \in \mathfrak{m}$ and $\Delta u=\sum_{i} a_{i} \otimes b_{i}$, then $L_{g} \bar{u}=$ $\sum a_{i}\left(g^{-1}\right) b_{i} \bmod \mathfrak{m}_{g}^{2}$. Hence

$$
L_{g} \bar{x}_{i}=\tilde{x}_{i}+\tilde{x}_{i}\left(g^{-1}\right) \tilde{z}_{i}^{-1} \in \mathfrak{m}_{g}
$$

so that $\tilde{x}_{i}(g)+\tilde{x}_{i}\left(g^{-1}\right) \tilde{z}_{i}(g)^{-1}=0$, and substituting in the above formula, we obtain

$$
L_{g} \bar{x}_{i}=\tilde{x}_{i}-\tilde{z}_{i}^{-1}\left(\tilde{x}_{i} \tilde{z}_{i}\right)(g)=\tilde{z}_{i}^{-1} d\left(\tilde{z}_{i} \tilde{x}_{i}\right) \quad \bmod \mathrm{m}_{g}^{2},
$$

(since $d u=u-u(g) \bmod \mathrm{m}_{g}^{2}$ ). We conclude that

$$
\widetilde{P}_{\tilde{z}_{i} \tilde{x}_{i}}=-d_{i} z_{i}\left(f_{i}, f_{i}\right) \text {. }
$$

On the other hand, Theorem 5.4 can be rewritten as

$$
P_{z_{i} x_{i}}=-d_{i} z_{i} f_{i}
$$

(here the normalization of $\S 7.3$ is essential). This proves that $\varphi^{*} \circ \widetilde{P}_{\tilde{z}_{i} \tilde{x}_{i}}=$ $P_{z_{i} x_{i}} \circ \varphi$. Similarly, we prove that $\varphi^{*} \circ \widetilde{P}_{\tilde{y}_{i}}=P_{y_{i}} \circ \varphi$ and $\varphi^{*} \circ \widetilde{P}_{\tilde{z}_{\alpha}}=P_{z_{\alpha}} \circ \varphi$ using Theorem 5.5 and Remark 5.5(a) respectively.

In order to complete the proof of (a), it remains to show that the elements $\tilde{x}_{i}, \tilde{y}_{i}, \tilde{z}_{\alpha}$ generate $\mathbb{C}[\widetilde{\Omega}]$ as a Poisson algebra. Note that $\mathbb{C}[\widetilde{\Omega}]=\mathbb{C}\left[U_{-}\right] \otimes$ $\mathbb{C}[T] \otimes \mathbb{C}\left[U_{+}\right]$, where $U_{ \pm}$and $T$ are embedded in $\widetilde{\Omega}$ in a natural way as Poisson algebraic subgroups. Hence it suffices to show that the $\tilde{x}_{i}$ (resp. $\tilde{y}_{i}$ ) generate $\mathbb{C}\left[U_{+}\right]$(resp. $\left.\mathbb{C}\left[U_{-}\right]\right)$as a Poisson subalgebra. For this note that $\mathbb{C}\left[U_{+}\right]$ is $\mathbb{Z}_{+}$-graded by $\operatorname{deg} x_{i}=1$. Let $\widetilde{\mathfrak{m}}_{+}$be its augmentation ideal. In order to show that the $\tilde{x}_{i}$ generate $\mathbb{C}\left[U_{+}\right]$as a Poisson subalgebra, it suffices to show that $\tilde{\mathfrak{m}}_{+} / \tilde{\mathfrak{m}}_{+}^{2}$ is generated as a Lie algebra by the $\bar{x}_{i}$. But this is clear since $\tilde{\mathfrak{m}}_{+} / \tilde{\mathfrak{m}}_{+}^{2}$ is the subalgebra $\mathfrak{n}_{+}$in the Lie algebra $\tilde{\mathfrak{m}} / \tilde{\mathfrak{m}}_{+}^{2} \simeq \mathfrak{g}$, the $\bar{x}_{i}$ corresponding to the $e_{i}$. This completes the proof of (a).

(b) follows from (a) and Theorem 4.7. 
7.7. Remarks. (a) The orbits of $\widetilde{G}$ on $\Omega_{M}$ are precisely the symplectic leaves of the Poisson structure on $\Omega_{M}$.

(b) Recall that, by Theorem 6.7(a) we have canonical isomorphisms $T / \widetilde{W} \simeq$ $\operatorname{Spec}\left(Z_{0}^{0 \widetilde{W}}\right) \simeq \operatorname{Spec}\left(Z_{0}^{\widetilde{G}}\right)$. Here $Z_{0}^{\widetilde{G}}$ is the ring of $\widetilde{G}$-invariant regular functions on $\Omega_{M}=$ the ring of regular functions on $\Omega_{M}$ constant along the symplectic leaves. Thus, the inclusion $Z_{0}^{\widetilde{G}} \hookrightarrow Z_{0}$ gives a map $\sigma: \Omega_{M} \rightarrow T / \widetilde{W}$. On the other hand, we have the $l$ th power map $\rho_{l}: T / \widetilde{W} \rightarrow T / \widetilde{W}$. Consider the fiber product $Y_{M}:=T / \widetilde{W} \times_{T / \widetilde{W}} \Omega_{M}$. We extend the Poisson structure from $\Omega_{M}$ to $Y_{M}$ trivially. We have the canonical map Spec $Z_{M, \varepsilon} \rightarrow Y_{M}$ and due to $\S 6.4$ this map is an isomorphism if $M=P$. Thus, according to Theorem 7.6, Spec $Z_{P, \varepsilon}$ as a Poisson variety can be constructed entirely in terms of the Poisson algebraic group $\widetilde{\Omega}_{P}$.

(c) For an $n$-tuple $\beta=\left(\beta_{1}, \ldots, \beta_{n}\right)$ of elements of $M$ one can associate a comultiplication $\Delta_{\beta}$ of $U_{M}$ by the formula

$$
\begin{aligned}
\Delta_{\beta} E_{i} & =E_{i} \otimes K_{\beta_{i}}+K_{\alpha_{i}-\gamma_{i}} \otimes E_{i}, \\
\Delta_{\beta} F_{i} & =F_{i} \otimes K_{-\alpha_{i}-\beta_{i}}+K_{\gamma_{i}} \otimes F_{i}, \\
\Delta_{\beta} K_{\alpha} & =K_{\alpha} \otimes K_{\alpha},
\end{aligned}
$$

where the $\gamma_{i} \in M$ are defined by equations $\left(\gamma_{i} \mid \alpha_{j}\right)=-\left(\beta_{j} \mid \alpha_{i}\right), i, j=$ $1, \ldots, n$. The Lie comultiplication on $\mathrm{m} / \mathrm{m}^{2}$ is then

$$
\delta \alpha_{i}=0, \quad \delta E_{i}=\left(\alpha_{i}-\beta_{i}-\gamma_{i}\right) \wedge E_{i}, \quad \delta F_{i}=\left(\alpha_{i}+\beta_{i}+\gamma_{i}\right) \wedge E_{i}
$$

The corresponding Manin triple is $\left(\mathfrak{g} \oplus \mathfrak{g}\right.$, Lie $\widetilde{\Omega}_{\beta}$, Lie $\left.K\right)$, where Lie $\widetilde{\Omega}_{\beta}=$ $\left(\mathfrak{n}_{-}, \mathfrak{n}_{+}\right)+\sum_{i} \mathbb{C}\left(-\alpha_{i}+\beta_{i}+\gamma_{i}, \alpha_{i}+\beta_{i}+\gamma_{i}\right)$. Theorem 7.6 holds in this more general situation.

NOTE ADDED IN PROOF

1. One may replace throughout the paper the condition $\left(l, d_{i}\right)=1$ for all $i$ by $l>d_{i}$ for all $i$ ( $l$ odd). This takes care of $\left(a_{i j}\right)$ of type $G_{2}$ and (odd) $l$ divisible by 3 . Then all results of the present paper still hold (with a little modification of their proofs). The same is true for [DCK] except that Theorem 3.2 and Corollary 3.2 should be modified. Consequently, the maximal dimension of an irreducible representation of $U_{\varepsilon}$ of type $G_{2}$ in the case when 3 divides $l$ is $l^{6} / 27$ (instead of $l^{6}$ when 3 does not divide $l$; see [DCK, Theorem 3.8]).

2. The case of $l$ divisible by 4 has been worked out recently by Jonathan Beck in his MIT thesis.

3. We can prove now the second part of Conjecture 6.8: if $\mathscr{O}$ is an orbit of maximal dimension, then $\operatorname{dim} V=l^{N}$. 


\section{REFERENCES}

[BC] P. Bala and R. W. Carter, Classes of unipotent elements in simple algebraic groups, II, Math. Proc. Cambridge Philos. Soc. 80 (1976), 1-18.

[D] V. G. Drinfeld, Quantum groups, Proc. ICM Berkeley 1 (1986), 789-820.

[DCK] C. De Concini and V. G. Kac, Representations of quantum groups at roots of 1 , Colloque Dixmier 1989, pp. 471-506; Progress in Math., vol. 92, Birkhäuser, Boston, MA, 1990.

[DCK1] _ Representations of quantum groups at roots of 1: reduction to the exceptional case, RIMS 792 preprint, 1991.

[KP] V. G. Kac and D. H. Peterson, Defining relations of certain infinite-dimensional groups, Astérisque, hors série (1985), 165-208.

[KW] V. G. Kac and B. Yu. Weisfeiler, Coadjoint action of a semi-simple algebraic group and the center of the enveloping algebra in characteristic $p$, Indag. Math. 38 (1976), 136-151.

[K] B. Kostant, Lie group representations on polynomial rings, Amer. J. Math. 86 (1963), 327402.

[L] G. Lusztig, Quantum groups at roots of 1, Geom. Dedicata 35 (1990), 89-114.

[LuR] J.-H. Lu and T. Ratiu, On the nonlinear convexity theorem of Kostant, J. Amer. Math. Soc. 4 (1991), 349-363.

[STS] M. A. Semenov-Tian-Shansky, Dressing transformations and Poisson group actions, Publ. RIMS 21 (1985), 1237-1260.

[Se] J.-P. Serre, Algebre locale. Multiplicites, Lecture Notes in Math., vol. 11, Springer-Verlag, Berlin-New York, 1975.

[S] R. Steinberg, Regular elements of semi-simple algebraic groups, Publ. Math. IHES 25 (1965), 49-80.

[WK] B. Yu. Weisfeiler and V.G. Kac, On irreducible representations of Lie p-algebras, Funct. Anal. Appl. 5 (1971), 28-36.

Scuola Normale Superiore, Pisa, Italy

Department of Mathematics, Massachusetts institute of Technology, Cambridge, MASSACHUSETTS 02139 ITALY

Dipartmento di Matematica, Universita Degli Studi di Roma "La Sapienza", Rome, 